\title{
ICOS signaling limits regulatory $T$ cell accumulation and function in visceral adipose tissue
}

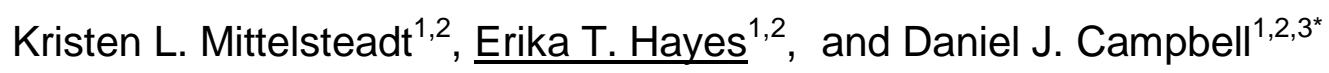 \\ ${ }^{1}$ Center for Fundamental Immunology, Benaroya Research Institute, Seattle, WA 98101 \\ ${ }^{2}$ Molecular and Cellular Biology Program, University of Washington, Seattle, WA 98195 \\ ${ }^{3}$ Department of Immunology, University of Washington, Seattle, WA 98195 \\ *Corresponding Author: Daniel J. Campbell, Benaroya Research Institute, 1201 Ninth \\ Avenue, Seattle, WA 98101. Email address: dcampbell@benaroyaresearch.org \\ Running Title: ICOS signaling in VAT- $\mathrm{T}_{\mathrm{R}}$
}

Summary: The authors demonstrate that loss of ICOS-dependent PI3-kinase signaling supports visceral adipose tissue (VAT) $T_{R}$ abundance and function, and this correlates with reduced adipose inflammation and improved insulin sensitivity after high-fat diet. This work highlights a new molecular pathway that regulates VAT- $T_{R}$ accumulation and activity. 


\begin{abstract}
A unique population of Foxp3 ${ }^{+}$regulatory T cells $\left(T_{R}\right)$ resides in visceral adipose tissue (VAT) that regulates adipose inflammation and helps preserve insulin sensitivity. The costimulatory molecule ICOS is highly expressed on effector $(e) T_{R}$ that migrate to nonlymphoid tissues, and contributes to their maintenance and function in models of autoimmunity. In this study, we report an unexpected cell-intrinsic role for ICOS expression and downstream PI3K signaling in limiting the abundance, VAT-associated phenotype, and function of $\mathrm{T}_{\mathrm{R}}$ specifically in VAT. I $\cos ^{-1-}$ mice and mice expressing a knock-in form of ICOS that cannot activate PI3K had increased VAT- $T_{R}$ abundance and elevated expression of canonical VAT-T $T_{R}$ markers. Loss of ICOS signaling facilitated enhanced accumulation of $\mathrm{T}_{\mathrm{R}}$ to VAT associated with elevated CCR3 expression, and resulted in reduced adipose inflammation and heightened insulin sensitivity in the context of high-fat diet. Thus, we have uncovered a new and surprising molecular pathway that regulates VAT- $T_{R}$ accumulation and function.
\end{abstract}




\section{Introduction}

$\mathrm{CD}^{+} \mathrm{Foxp}^{+}$regulatory $\mathrm{T}$ cells $\left(\mathrm{T}_{\mathrm{R}}\right)$ are critical for maintaining immune tolerance and for the resolution of ongoing inflammation after infection (Dominguez-Villar and Hafler, 2018; Rivas and Chatila, 2016; Smigiel et al., 2014a). Specialized subsets of tissue-specific $T_{R}$ also function in tissue repair and homeostasis. For example, $\mathrm{Areg}^{+} \mathrm{T}_{R}$ expand in skeletal muscle and the lungs in response to injury and are required for proper tissue repair (Arpaia et al., 2015; Burzyn et al., 2013), whereas $T_{R}$ within hair follicles and skin are essential for hair generation and inhibiting fibrosis (Ali et al., 2017; Kalekar et al., 2019). $T_{R}$ are also found in the visceral adipose tissue (VAT) of both mice and humans, where they regulate adipose inflammation and preserve metabolic homeostasis (Deiuliis et al., 2011; Eller et al., 2011; Feuerer et al., 2009; llan et al., 2010). As such, $T_{R}$ ablation in diphtheria toxin-treated Foxp $3^{\mathrm{DTR}}$ mice results in increased inflammatory mediators in VAT and reduced insulin sensitivity, whereas augmentation of $\mathrm{T}_{\mathrm{R}}$ by administration of IL-2 immune complexes or IL-33 results in improved metabolic readouts in mice on high-fat diet (HFD) (Feuerer et al., 2009; Han et al., 2015; Vasanthakumar et al., 2015).

Given that $T_{R}$ modulate diverse responses in different anatomical and inflammatory settings, it is not surprising that they exhibit considerable phenotypic and functional heterogeneity. Expression of CD62L and CD44 broadly divides $T_{R}$ into distinct subsets that are enriched in lymphoid and nonlymphoid tissues (Campbell, 2015; Smigiel et al., 2014b). We term these populations central $T_{R}\left(c T_{R}\right)$ and effector $T_{R}$ $\left(\mathrm{e} \mathrm{T}_{\mathrm{R}}\right)$, respectively. Differential activation of $\mathrm{PI} \mathrm{K}$, unique epigenetic landscapes, and distinct transcriptional programs underlie the diversification of $T_{R}$, and selective expression of chemokine receptors and cell adhesion molecules give subsets of $T_{R}$ access to specific tissues (Delacher et al., 2017; DiSpirito et al., 2018; Luo et al., 2016). The proper distribution of $T_{R}$ across tissues is crucial for maintaining immune tolerance and tissue homeostasis (Sather et al., 2007; Yamaguchi et al., 2011). Hence, understanding the signals that control the development, maintenance, and function of tissue-specific $T_{R}$ is vital to fully harnessing their therapeutic potential.

$T_{R}$ occupancy in different tissues is met with unique homeostatic maintenance requirements. Generally, IL-2 signaling maintains $\mathrm{CT}_{\mathrm{R}}$ within $\mathrm{T}$ cell zones of secondary lymphoid tissues by driving pro-survival signals, whereas maintenance of $\mathrm{eT}_{\mathrm{R}}$ in nonlymphoid tissues can be IL-2 independent and instead relies on TCR and costimulatory molecule engagement (Levine et al., 2014; Smigiel et al., 2014b; Tang et al., 2003). ICOS is expressed on highly suppressive $T_{R}$ and can control $T_{R}$ abundance by inhibiting apoptosis and stimulating proliferation (Burmeister et al., 2008; Redpath et al., 2013). IL-10 is essential for control of local immune responses, and is primarily expressed by Blimp- $1^{+} \mathrm{ICOS}^{+} \mathrm{eT}_{\mathrm{R}}$ (Cretney et al., 2011). ICOS costimulation in vitro super-induces IL-10 expression (Arimura et al., 2002; Hutloff et al., 1999), and this depends on its ability to activate phosphoinositide 3-kinase (PI3K) (Feito et al., 2003; Okamoto et al., 2003). Although mice deficient in ICOS or ICOS ligand do not develop spontaneous inflammatory or autoimmune disease, genetic or antibody-mediated blockade of ICOS signaling promotes inflammation in mouse models of autoimmunity and infection as a result of reduced $\mathrm{T}_{\mathrm{R}}$ abundance and/or IL-10 production (Busse et al., 2012; Dong et al., 2001; Herman et al., 2004; Kohyama et al., 2004; Kornete et al., 2012; Landuyt et al., 2019; Miyamoto et al., 2005; Redpath et al., 2013). 
ICOS potently activates PI3K and downstream AKT via a unique YMFM motif in its cytoplasmic tail (Arimura et al., 2002; Fos et al., 2008). AKT can act on several different substrates, including the transcription factor Foxo1, to modulate transcription of genes involved in proliferation, survival, metabolic reprogramming, and migration/tissue tropism (So and Fruman, 2012). Indeed, inactivation of Foxo1 by AKT is essential for the differentiation of $\mathrm{eT}_{\mathrm{R}}$ and their migration to nonlymphoid tissues (Luo et al., 2016). The mammalian target of rapamycin (mTOR) pathway is also activated by PI3K signaling, and regulates Treg cell metabolism and differentiation. Thus, engagement of ICOS may facilitate the development and/or maintenance of $\mathrm{eT}_{\mathrm{R}}$ via multiple PI3Kdependent signaling pathways.

Accumulation of $T_{R}$ in VAT is dependent on several signals. VAT- $T_{R}$ consist of clonally expanded populations, suggesting recognition of one or more adipose tissue antigens promotes their tissue residence (Feuerer et al., 2009; Kolodin et al., 2015). Additionally, mice lacking expression of IL-33 or its receptor ST2 are deficient in VAT$T_{R}$, whereas injection of exogenous IL-33 results in increased VAT- $T_{R}$ abundance with little impact on lymphoid $T_{R}$ (Han et al., 2015; Vasanthakumar et al., 2015). Recent work also identified a cell-intrinsic role for CCR2 in the ability of donor $T_{R}$ to repopulate VAT (Vasanthakumar et al., 2020). Finally, $T_{R}$ expression of PPARy, the master regulator of adipocyte differentiation, supports VAT- $\mathrm{T}_{\mathrm{R}}$ phenotype and accumulation and drives expression of factors important for lipid metabolism (Cipolletta et al., 2012; Cipolletta et al., 2015). However, despite having an $\mathrm{eT}_{\mathrm{R}}$ phenotype, the role of costimulatory molecule signaling in VAT- $T_{R}$ is poorly understood.

In this study, we demonstrate an unexpected role for cell-intrinsic ICOSdependent PI3K signaling in restricting VAT- $\mathrm{T}_{\mathrm{R}}$ accumulation and function. Moreover, we implicate the CCL11/24-CCR3 axis as an additional factor capable of driving recruitment of $T_{R}$ to VAT, which is enhanced in the absence of ICOS signaling. These surprising findings challenge the current model regarding signals that support diverse $T_{R}$ subsets, and highlight the cell- and tissue-specific effects of ICOS expression and signaling on $T_{R}$ development, accumulation, and function.

\section{Results}

Mice lacking ICOS signaling have reduced $T_{R}$ in lymphoid tissues and altered expression of PI3K targets

To determine how ICOS signaling impacts $T_{R}$ abundance in different tissues, we crossed Foxp3 ${ }^{\text {mRFP }}$ mice to $I \cos ^{-1-}$ (KO) mice and to $I \cos ^{Y 181 F}$ (YF) mice, which carry a tyrosine-to-phenylalanine knock-in mutation in the YMFM motif in the cytoplasmic tail of ICOS, thereby specifically abolishing ICOS-mediated PI3K activation (Gigoux et al., 2009), to Foxp3 ${ }^{\text {RRFP }}$ mice (Wan and Flavell, 2005). In line with published data, we found a $\sim 30 \%$ reduction in the frequency and number of $T_{R}$ in the spleens of $K O$ compared to WT Foxp3 ${ }^{\text {mRFP }}$ mice (Burmeister et al., 2008; Landuyt et al., 2019) [Fig. 1A]. This was even more pronounced in older animals, as $T_{R}$ accumulate with age (Nishioka et al., 2006) [Fig. 1B]. Despite normal ICOS expression on the cell surface [Fig. S1 A] and intact MAPK signaling (Gigoux et al., 2009), we saw a similar reduction in splenic $T_{R}$ abundance in YF mice, indicating that activation of $\mathrm{PI} 3 \mathrm{~K}$ signaling is the critical pathway by which ICOS regulates $T_{R}$ abundance [Fig. 1A, 1B]. ICOS is expressed on ${ }^{2} T_{R}$, but its expression is further upregulated on $\mathrm{e}_{R}$ [Fig. S1B]. The loss of $T_{R}$ we observed in 
YF and $\mathrm{KO}$ spleens was associated with a disproportionate reduction in the frequency and number of $\mathrm{e} \mathrm{T}_{\mathrm{R}}$, whereas $\mathrm{CT}_{\mathrm{R}}$ abundance was unchanged, consistent with results using antibody blockade of ICOS signaling (Smigiel et al., 2014b) [Fig. 1C]. Loss of $T_{R}$ was also observed in the peripheral lymph nodes ( $\mathrm{pLN}$ ) of YF and KO mice, indicating that ICOS signaling is important for $\mathrm{eT}_{\mathrm{R}}$ maintenance in multiple secondary lymphoid organs [Fig. S1C]. Both YF and $K O T_{R}$ in the spleen had elevated expression of the lymphoid homing molecules CD62L and CCR7 which are downregulated upon PI3K activation via phosphorylation and sequestration of Foxo1 (Kerdiles et al., 2009) [Fig. 1D]. This reflects both an increased frequency of splenic $c T_{R}$ as well as increased CD62L and CCR7 expression by gated CD44 ${ }^{\mathrm{hi}} \mathrm{T}_{\mathrm{R}}$ in YF and KO mice..When examined directly ex vivo, $\mathrm{YF}$ and $\mathrm{KO}$ splenic $\mathrm{T}_{\mathrm{R}}$ also exhibited reduced phosphorylation of the mTORC1 target, ribosomal protein S6, consistent with diminished activation of PI3K [Fig. 1E]. Thus, the absence of ICOS-mediated PI3K activation drives the altered lymphoid $T_{R}$ frequency and phenotype we see in YF and $K O$ mice.

\section{$T_{R}$ are enriched in VAT in the absence of ICOS signaling}

As $\mathrm{eT}_{\mathrm{R}}$ are highly enriched in nonlymphoid sites (Lee et al., 2007; Smigiel et al., 2014b), we next assessed whether maintenance of $T_{R}$ in peripheral tissues was diminished in YF and KO mice. Using intravascular labeling to identify tissue-localized $T_{R}$ (Anderson et al., 2014), we observed a reduction in the frequency and number of $T_{R}$ in the skin and decrease in the absolute number of $T_{R}$ in the lungs of $Y F$ and $K O$ mice [Fig. 2A, 2B]. However, $T_{R}$ frequency and total number were actually elevated in the VAT of both strains [Fig. 2C]. This was specific to VAT, as frequency and total number of $T_{R}$ in subcutaneous adipose tissue (SQAT) remained similar between genotypes [Fig. 2D]. Male and female YF and KO mice demonstrated increased VAT- $T_{R}$ abundance compared to WT mice of the same sex, however the frequency and number of VAT- $T_{R}$ in females was significantly less than that observed in males [Fig. 2E, 2F]. Consistent with previous studies, we observed age-dependent accumulation of VAT- $T_{R}$ across all three genotypes in male mice (Feuerer et al., 2009), but despite considerable mouse-to-mouse variability, VAT- $\mathrm{T}_{\mathrm{R}}$ remained elevated in YF and KO mice across all age groups analyzed [Fig. 2G].

We utilized male mice for the remainder of our studies as we found female mice harbor $\sim 4-5$-fold fewer $\mathrm{T}_{\mathrm{R}} /$ gram VAT than age-matched males, and female mice are protected from the development of diet-induced metabolic syndrome (Ahnstedt et al., 2018; Pettersson et al., 2012; Vasanthakumar et al., 2020). The frequency of VAT- $T_{R}$ expressing KLRG1 and CD69 was significantly elevated in YF and KO mice compared to WT controls [Fig. 3A]. By contrast, in the spleen there was a reduced frequency of $Y F$ and $K O T_{R}$ expressed CD69, and very few $T_{R}$ were $K L R G 1^{+}$[Fig. S2A]. We assessed VAT- $T_{R}$ Foxp3 and CD44 expression, and found no difference in the absence of ICOS [Fig. S2B]. We did however observe a modest increase in the expression of CTLA-4 and a slight reduction in the frequency of CD25 ${ }^{+} T_{R}$ in YF and KO VAT [Fig. 3A]. Therefore, $T_{R}$ in the VAT of $Y F$ and $K O$ mice are enriched in expression of markers indicative of an $\mathrm{eT}_{\mathrm{R}}$ and VAT- $\mathrm{T}_{\mathrm{R}}$ phenotype (Feuerer et al., 2009; Smigiel et al., 2014b).

In addition to prototypical e $T_{R}$ markers, a fraction of VAT- $T_{R}$ express the IL-33 receptor, ST2 (Kolodin et al., 2015; Vasanthakumar et al., 2015). Signaling downstream of ST2 is required for the establishment and maintenance of VAT- $T_{R}$, and expansion of 
VAT- $T_{R}$ through administration of IL-33 can reduce VAT inflammation and improve metabolic indices in models of diet-induced obesity (Han et al., 2015; Kolodin et al., 2015; Vasanthakumar et al., 2015). In addition to ST2-expressing $T_{R}$, we found a substantial population of $\mathrm{CXCR}^{+} \mathrm{T}_{\mathrm{R}}$ in the VAT of lean mice, confirming previously reported findings [Fig. 3B] (Li et al., 2018). Although there were no significant differences in the frequency of WT, YF, or KO VAT- $T_{R}$ singly-expressing ST2 or CXCR3, we found a unique population of double-expressing $S T 2^{+} C X C R 3^{+} T_{R}$ in $Y F$ and KO VAT which resembled canonical VAT- $T_{R}$ in their expression of KLRG1 and CD69 [Fig. 3B, 3C]. Furthermore, whereas the abundance of splenic Treg cells in WT mice was dramatically reduced following treatment with the mTORC1 inhibitor rapamycin, there was no significant change in the numbe of Treg cells in the VAT [Fig. S3], indicating that ICOS-dependent PI3K activation regulates VAT Treg cells independent of mTORC1 activity.

ICOS signaling can potentiate IL-10 production (Busse et al., 2012; Dong et al., 2001; Herman et al., 2004; Hutloff et al., 1999; Kohyama et al., 2004; Kornete et al., 2012; Landuyt et al., 2019; Miyamoto et al., 2005; Redpath et al., 2013). Surprisingly, compared to VAT- $T_{R}$ from WT mice, a substantially higher frequency of $\mathrm{YF}$ and KO VAT- $T_{R}$ were poised to produce IL-10 upon ex vivo stimulation with PMA/I, with no appreciable differences in IL-10 expression by splenic $T_{R}$ [Fig. 3D]. Thus, absence of ICOS-dependent PI3K signaling supports both the accumulation and suppressive phenotype of $T_{R}$ in VAT.

\section{Baseline adipose inflammation is reduced in YF and $K O$ mice}

VAT inflammation is modulated through a balance of different immune cells. $T_{R}$, eosinophils, anti-inflammatory adipose tissue macrophages (ATM), and group-2 innate lymphoid cells (ILC2) maintain a type-2 immune environment that supports metabolic homeostasis in lean animals. Inflammation however drives recruitment of neutrophils and cytotoxic T cells, differentiation of inflammatory ATM, and production of proinflammatory cytokines like TNFa (Burhans et al., 2018; Molofsky et al., 2013). Although there was no difference in mouse weights between WT, YF, and KO mouse across different ages, we did observe a trend toward increased VAT weight in YF mice, and a significant increase in $\mathrm{KO}$ mice [Fig. 4A]. By histology, we did not observe any overt morphological differences in VAT from WT, YF, and KO mice, including no observable differences in adipocyte size [Fig. S4A]. The increase in VAT weight however did correlate with a modest increase in the total number of $\mathrm{CD} 4^{+} \mathrm{T}_{\text {eff }}$ per gram of tissue [Fig. 4B]. Despite this increase in VAT- $T_{\text {eff }}$ number, there was a substantially lower frequency of $Y F$ and KO VAT- $T_{\text {eff }}$ expressing features of inflammatory $T_{H} 1$ cells, including CXCR3, IFNy, and TNFa [Fig. 4C]. Rather, ST2-expressing $T_{\text {eff }}$ were enriched in YF and KO VAT [Fig. 4C]. Similar to VAT- $T_{R}$, YF and KO VAT- $T_{\text {eff }}$ were superior at producing IL-10 upon stimulation, suggesting that absence of ICOS signaling in effector cells supports a self-regulatory function [Fig. 4C]. IL-33 signaling induces production of $\mathrm{T}_{\mathrm{H}} 2$ cytokines (Schmitz et al., 2005), which maintain ATM in an anti-inflammatory state. While we didn't find any significant changes in ATM or ILC2 populations [Fig. S4B, 4C], we did note an increased frequency of eosinophils in YF and KO VAT [Fig. 4D], which produce IL-4 to maintain anti-inflammatory ATM (Molofsky et al., 2013; Wu et al., 2011). 
Overall, global loss of ICOS expression and signaling impacts $T_{R}$ along with other adipose-resident immune cells to maintain an anti-inflammatory state in VAT.

\section{YF and KO mice are protected from high-fat diet-induced insulin resistance}

Given the abundance and suppressive phenotype of $\mathrm{T}_{\mathrm{R}}$ and other antiinflammatory immune cells in VAT in YF and KO mice, we asked if they were more resistant to developing HFD-induced adipose inflammation and metabolic changes. Mice placed on an 18-week HFD steadily gained body mass, with a modest reduction in weight gained by KO mice compared to WT [Fig. 5A]. After 18 weeks on HFD, YF and KO mice sustained elevated $T_{R}$ frequencies in VAT compared to WT mice, with an increased fraction of cells expressing the canonical VAT- $T_{R}$ markers ST2 and KLRG1, and a smaller proportion of $T_{R}$ expressing CXCR3 [Fig. 5B, 5C]. Additionally, YF and $\mathrm{KO}$ mice placed on HFD retained a greater frequency of eosinophils and $\mathrm{T}_{\text {eff }}$ skewed toward a type-2, ST2-expressing phenotype [Fig. 5D, 5E]. Fasting blood glucose levels (BGL) were similar among genotypes after 18-weeks, and WT, YF, and KO mice were able to clear glucose to the same extent, as measured by glucose tolerance test (GTT) [Fig. 5F]. However, in an insulin tolerance test (ITT), YF and KO mice demonstrated improved insulin sensitivity compared to WT mice [Fig. 5G]. Thus, loss of ICOS supports $T_{R}$ and other anti-inflammatory cells in VAT during HFD, and this has functional consequences in maintaining metabolic homeostasis.

\section{Absence of ICOS supports the accumulation and phenotype of VAT-TR in a cell-intrinsic manner}

ICOS signaling supports a wide array of cellular processes in the context of adaptive immunity (Panneton et al., 2019). To determine which of the T cell phenotypes we observed in intact YF and KO mice were the result of cell-intrinsic ICOS expression and signaling, we generated mixed bone marrow chimeras using congenically marked CD45.1 WT and CD45.2 WT, YF, or KO donors [Fig. 6A]. Body and adipose depot weights were similar between groups of chimeric mice [Fig. S5A]. Consistent with the increased abundance of VAT- $T_{R}$ we observed at baseline in intact $Y F$ and KO mice, both CD45.2 $2^{+} \mathrm{YF}$ and $\mathrm{KO} \mathrm{T}_{\mathrm{R}}$ displayed a competitive advantage compared to CD45. $1^{+}$ WT $T_{R}$ in repopulating VAT in our chimeric mice. While WT:WT chimeras reconstituted VAT- $T_{R}$ at $a \sim 1: 1$ ratio, $Y F$ and $K O T_{R}$ exhibited a significantly higher reconstitution ratio of $~ 5.5: 1$ [Fig. 6B]. In addition to supporting VAT- $T_{R}$ abundance, absence of cellintrinsic ICOS signaling also promoted a canonical VAT- $T_{R}$ phenotype. Within the same chimeras, a higher frequency of YF and KO VAT- $T_{R}$ expressed CD69 and ST2 [Fig. 6C]. Aligned with data from intact mice, more $Y F$ and KO VAT- $T_{R}$ within chimeras were double-expressers of CXCR3 and ST2 [Fig. 6C]. Functionally, a larger proportion of YF and KO VAT- $T_{R}$ were poised to produce IL-10 after ex vivo stimulation [Fig. 6C]. Mixed bone marrow chimeras retain a population of radioresistant host $T_{R}$, which proliferate to replenish a portion of the available niche after irradiation (Komatsu and Hori, 2007), and this is particularly evident in many nonlymphoid tissues. Remarkably, in addition to preferentially reconstituting VAT compared to WT donor $T_{R}$, YF and KO VAT- $T_{R}$ were able to outcompete endogenous WT VAT- $T_{R}$ as well, nearly replacing the entire $T_{R}$ compartment in some chimeras [Fig. 6D]. 
Contrary to our findings in VAT, YF and $K O \mathrm{~T}_{\mathrm{R}}$ were at a selective disadvantage in the skin, spleen, and large intestine, with no difference in reconstitution in the lungs [Fig. 6E, Fig. S5B]. $T_{R}$ found in SQAT are phenotypically distinct from VAT- $T_{R}$ and do not appear to accumulate with age (Feuerer et al., 2009; Li et al., 2018). However, unlike in intact mice, we did note that $\mathrm{YF}$ and $\mathrm{KO}$ donor $\mathrm{T}_{\mathrm{R}}$ displayed a competitive advantage in repopulating SQAT in chimeric mice at a ratio of $\sim 3: 1$ [Fig. 6E]. This could be due to low level inflammation following irradiation of the recipient mice that drives reconstitution of the SQAT niche with $T_{R}$ exhibiting a more VAT- $T_{R}$ phenotype.

Although $\mathrm{CD} 8^{+} \mathrm{T}$ cells and ILC2s of either genotype were similarly represented [Fig. S5C], we did find that YF and KO Teff were modestly, but significantly, enriched in VAT, at a $\sim 2: 1$ ratio compared to WT $T_{\text {eff }}$ with no difference in reconstitution of other tissues [Fig. 6F]. A higher frequency of $\mathrm{YF}$ and KO VAT- $\mathrm{T}_{\text {eff }}$ were ST2 ${ }^{+}$within the same chimeras, with a reduction in CXCR3-expressing $T_{\text {eff }}$ [Fig. 6G]. Functionally, YF and KO VAT- $\mathrm{T}_{\text {eff }}$ were comprised of fewer IFNy ${ }^{+}$and $\mathrm{TNFa}^{+}$and more IL- $10^{+}$cells when stimulated ex vivo [Fig. 6G], indicating that in addition to driving increased $T_{R}$ abundance, the absence of ICOS signaling also promotes a regulatory phenotype in VAT- $T_{\text {eff. }}$ Taken together, loss of ICOS signaling supports VAT- $T_{R}$, and to a lesser extent, $T_{\text {eff }}$ accumulation and phenotype through cell-intrinsic mechanisms.

\section{ICOS signaling impacts expression of homing receptors in $T_{R}$ that allow access to VAT}

The increased abundance of VAT- $T_{R}$ from $Y F$ and $K O$ mice could result from the combination of increased proliferation, increased survival, or increased migration to the VAT. The proliferation marker Ki67 was expressed by $\sim 10-20 \%$ of VAT- $T_{R}$, and we found no significant difference in the frequency of Ki67 $7^{+} V A T-T_{R}$ between WT, YF, and KO mice [Fig. 7A]. Similarly, WT, YF, and KO VAT- $T_{R}$ expressed similar levels of the pro-survival protein Bcl2 [Fig. 7B]. Despite the lack of differences in Ki67 or Bcl-2 expression, we cannot rule out that changes in cellular turnover contribute to the differences we see, as $T_{R}$ express other pro-survival factors. However, recent data demonstrating continuous recruitment of $T_{R}$ to VAT via the circulation led us to assess potential differences in migration of cells (Vasanthakumar et al., 2020).

In both intact and mixed bone marrow chimeric mice, YF and KO splenic $T_{R}$ contained a small but significantly increased population of $\mathrm{ST}^{+} \mathrm{T}_{\mathrm{R}}$ [Fig. 8A, 8B], a subset of $T_{R}$ that is both transcriptionally and epigenetically poised to take up residence in peripheral tissues (Delacher et al., 2017; Delacher et al., 2020). Given this, we hypothesized that superior migration and accumulation could account for the increased frequency of VAT- $T_{R}$ in YF and KO mice. Although CCR2 facilitates recruitment of $T_{R}$ to VAT (Vasanthakumar et al., 2020), we detected no difference in CCR2 expression among genotypes, and a modest decrease in $\mathrm{CCR} 2^{+} \mathrm{T}_{\mathrm{R}}$ in $\mathrm{KO}$ spleens [Fig. S6A], arguing against enhanced CCR2-mediated recruitment of YF and KO $T_{R}$ to VAT.

Previous studies identified an open chromatin landscape around the Ccr3 locus

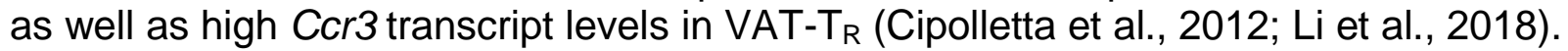
The frequency of CCR3-expressing splenic $T_{R}$ was slightly elevated in both young ( $\leq 8$ weeks) and older (>8 weeks) YF and KO mice [Fig. S6B], indicating the presence of a $T_{R}$ population primed for both potential early seeding and continuous repopulation of peripheral tissues. In line with this, YF and KO VAT contained more $C C R 3^{+} T_{R}$ compared to WT mice and this population increased with age [Fig. 8C]. In mixed bone 
marrow chimeras, a significantly greater frequency of $\mathrm{YF}$ and $\mathrm{KO}$ donor $\mathrm{T}_{\mathrm{R}}$ were $\mathrm{CCR} 3^{+}$ in spleen and VAT [Fig. S6C]. Additionally, YF and KO VAT contained a unique population of $T_{R}$ that expressed CCR3 alone, while WT VAT- $T_{R}$ expressing CCR3 were also positive for CCR2 [Fig. 8D]. CCR3 expression was unique to VAT-T $R$, unlike

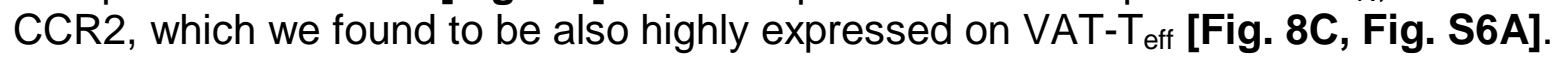
Additionally, the accumulation of $\mathrm{CCR} 3^{+} \mathrm{T}_{\mathrm{R}}$ was unique to VAT, as we detected very little CCR3 expression in skin, lungs, and large intestine $T_{R}$, and no differences across genotypes [Fig. S6D, S6E]. We detected substantial expression of the CCR3 ligands Ccl11 and Ccl24 in the VAT, with no differences detected either by age or genotype and little expression of Cc/5, Cc/7, and Ccl26 [Fig. 8E].

To determine if ICOS signaling could directly impact expression of CCR3 on $T_{R}$, WT, YF, and KO VAT-CD4 ${ }^{+}$cells were cultured with aCD3/aCD28 in the presence or absence of an agonistic alCOS antibody [Fig. 8F]. We additionally added $500 \mathrm{U} / \mathrm{mL} \mathrm{IL}-$ 2 to all conditions to account for any deficiencies in IL-2 production by YF and KO VAT$\mathrm{T}_{\text {eff. }}$ After $48 \mathrm{hr}$ in culture, $\mathrm{T}_{\mathrm{R}}$ frequency remained unchanged in both groups. However, the frequency of $\mathrm{CCR}^{+} \mathrm{T}_{\mathrm{R}}$ in WT cultures significantly decreased after 2 days with addition of an ICOS agonist, whereas no changes were observed in YF or KO cultures, indicating that ICOS-dependent PI3K signaling can directly antagonize CCR3 expression in $\mathrm{T}_{\mathrm{R}}$ [Fig. 8F].

To address the impact of CCR3 signaling on continuous $T_{R}$ seeding of VAT in vivo, we administered blocking antibodies to the CCR3 ligands CCL11 and CCL24 to 810 week old WT and YF mice. Blockade of CCL11/24 for 2 weeks resulted in a modest although not statistically significant decrease in the frequency of VAT- $T_{R}$ specifically in YF mice, with no differences in $T_{R}$ abundance in spleen or lungs between treated and untreated mice [Fig. 8G]. We additionally noted a slight reduction in VAT-eosinophils in YF mice treated with aCCL11/24 [Fig. S6F]. Together, these results suggest a potential mechanism by which loss of ICOS-dependent PI3K signaling can drive increased recruitment of $T_{R}$ to VAT by CCR3-CCL11/24 interactions.

\section{Discussion}

We have identified surprising tissue-specific effects of ICOS signaling on $T_{R}$ abundance and phenotype. Although there was a significant loss of $\mathrm{CD}_{4} 4^{+} \mathrm{eT}_{\mathrm{R}}$ in the spleen, pLN, skin, and lungs of YF and KO mice compared to WT, there was a substantial increase in $T_{R}$ abundance in VAT. $T_{R}$ frequency and phenotype were similar in YF and KO mice, including comparably altered readouts of PI3K signaling, suggesting that ICOS primarily impacts $\mathrm{T}_{\mathrm{R}}$ abundance and homeostasis via PI3Kdependent mechanisms.

In addition to supporting VAT- $T_{R}$ abundance, loss of ICOS signaling promoted a highly suppressive VAT- $T_{R}$ phenotype, including an increased proportion of $T_{R}$ expressing KLRG1, CD69 and IL-10. In vitro, addition of ICOS stimulation augments IL10 production in $C D 4^{+}$T cells while loss of ICOS signaling in vivo results in reduced $T_{R}$ abundance and IL-10 expression in models of autoimmunity, allergy, and infection (Busse et al., 2012; Hutloff et al., 1999; Kohyama et al., 2004; Kornete et al., 2012; Landuyt et al., 2019; Redpath et al., 2013). Although ICOS expression is associated with IL-10-producing $T_{R}$, ICOS signaling does not appear to be intrinsically required for $\mathrm{IL}-10$ production. CD $4^{+} \mathrm{T}$ cells from $\mathrm{KO}$ mice are capable of producing WT levels of IL- 
10 in vitro, and interestingly, appear to produce more IL-10 than WT counterparts under $T_{H}$ 2-polarizing conditions (Dong et al., 2001). Furthermore, recent studies assessing $T_{R}$ in the brain during chronic Toxoplasma gondii infection and lamina propria $\mathrm{T}_{\mathrm{R}}$ at baseline report no changes in IL-10 production in the presence or absence of ICOS signaling (Landuyt et al., 2019; O'Brien et al., 2019). Therefore, the reduction in IL-10+ $\mathrm{T}_{\mathrm{R}}$ with ICOS blockade observed in specific in vivo models is likely due to insufficient activation or access to specific environmental cues within tissues rather than an inherent requirement for ICOS signaling to drive IL-10 production.

VAT is a complex tissue, consisting of triglyceride-containing adipocytes and a variety of immune cells that function to maintain metabolic homeostasis. In YF and KO mice, other immune cells in addition to $T_{R}$ showed evidence of an anti-inflammatory phenotype. Although ATM and VAT-ILC2 abundance was similar across genotypes at baseline, eosinophil frequency was significantly elevated in YF and KO VAT. In YF and $\mathrm{KO}$ mice, VAT- $\mathrm{T}_{\text {eff }}$ were skewed toward a $\mathrm{T}_{\mathrm{H}} 2$ phenotype in a cell-intrinsic manner, with an increased frequency of ST2-expressing and IL-10-producing cells. Therefore, in addition to promoting an anti-inflammatory state by sustaining VAT- $T_{R}$, global loss of ICOS signaling supports the abundance and phenotype of other anti-inflammatory adipose-resident immune cells. These combined changes in cellular composition in VAT in the absence of ICOS signaling were maintained in the setting of long-term HFD, and rendered YF and KO mice more resistant to HFD-induced loss of insulin sensitivity. Changes in insulin responsiveness were subtle, despite a significant increase in $T_{R}$ in YF and KO mice. A recent study demonstrated that ablation of IL-10 improved insulin sensitivity in mice (Rajbhandari et al., 2018). Thus it is possible that the increased IL-10 production we observe in VAT- $T_{R}$ and VAT- $T_{\text {eff }}$ in the absence of ICOS signaling offsets the insulin sensitizing effects of increased VAT- $T_{R}$ abundance in these mice.

YF and $K O$ donor $T_{R}$ preferentially repopulated VAT in mixed bone marrow chimeras, and were able to outcompete both donor and endogenous WT $T_{R}$. ST2 is expressed on a large fraction of VAT- $T_{R}$ (Vasanthakumar et al., 2015), and although the frequency of ST2-expressing VAT- $T_{R}$ was not changed in intact mice, YF and KO VAT$\mathrm{T}_{\mathrm{R}}$ in mixed bone marrow chimeras were comprised of significantly more $\mathrm{ST}_{2}{ }^{+}$cells compared to WT donor $T_{R}$, suggesting that the competitive advantage of $\mathrm{YF}$ and $\mathrm{KO}$ donor $T_{R}$ in chimeric VAT is in part due to an enhanced ability to access available IL-33 through increased ST2 expression. The transcriptional regulators BATF and IRF4 cooperate to induce expression of ST2 in $T_{R}$, possibly downstream of TCR signals as well as through IL-33 signaling itself (Vasanthakumar et al., 2015). Additionally, IL-2 can work synergistically with IL-33 to upregulate ST2 and specifically expand ST2 ${ }^{+} \mathrm{T}_{\mathrm{R}}$ (Guo et al., 2009; Matta et al., 2014). $T_{R}$ are unable to produce IL-2 themselves due to Foxp3-mediated transcriptional repression at the II2 locus (Wu et al., 2006; Ono et al., 2007) and therefore are dependent on paracrine sources of IL-2 produced by, for example, autoreactive $T_{\text {eff }}$ (Setoguchi et al., 2005; Stolley and Campbell, 2016). However, KO $T_{\text {eff }}$ are defective in production of IL-2 compared to $T_{\text {eff }}$ from WT mice (Dong et al., 2001). The absence of an enriched population of ST2-expressing YF and KO VAT- $T_{R}$ at baseline may be explained by this lack of IL-2, which is rescued in the presence of WT IL-2 producing cells in mixed bone marrow chimeras.

Molofsky et al. have argued that IL-33 signaling on $\mathrm{T}_{\mathrm{R}}$ in VAT is mediated through ICOS/ICOSL interactions with ILC2s and that absence of this interaction results 
in loss of VAT- $T_{R}$ (Molofsky et al., 2015). In their system, blockade of ICOS signaling was assessed in $\mid \cos \Gamma^{--}$mice. Consistent with their data in $I \cos I^{-1}$ mice, we did not observe any differences in ILC2 frequency or number in VAT of KO or YF mice. However, given that ILC2s express both ICOS and ICOSL, loss of one versus the other could differentially impact ILC2 function, resulting in unique cell extrinsic impacts on $T_{R}$. Additionally, recent work revealed ligand-independent, constitutive ICOS signaling through PI3K-dependent pathways which relies on p85 association with the ICOS cytoplasmic YMFM motif and interactions between the ICOS transmembrane domain and the tyrosine kinase Lck (Feito et al., 2003; Wan et al., 2020). Thus the reported differences observed in our study and Molofsky's may be the result of low level, cell intrinsic PI3K activation in ICOS-expressing $\mathrm{T}_{\mathrm{R}}$ from $\mathrm{Icos}^{{ }^{-1}}$ mice.

Although CCR2 plays an important role in recruitment of $T_{R}$ to VAT, we did not detect any differences in CCR2 expression in the absence of ICOS signaling. However, the frequency of $\mathrm{CCR}^{+} \mathrm{T}_{\mathrm{R}}$ was increased in the spleen and VAT of YF and KO mice, which was due to cell-intrinsic loss of ICOS signaling and was enhanced with age. CCR3 is primarily associated with type-2 responses (Danilova et al., 2015; Francis et al., 2007; Humbles et al., 2002; Ma et al., 2002; Nagakubo et al., 2016), and lean, metabolically healthy VAT is maintained in an anti-inflammatory state by the presence of $\mathrm{T}_{\mathrm{H}} 2$-associated cells. In vivo blockade of CCL11/24 resulted in a modest reduction of $\mathrm{T}_{\mathrm{R}}$ in YF VAT with no changes in other tissues analyzed. Parabiosis experiments demonstrate recruitment of circulating parabiont-derived $T_{R}$ to VAT after months of shared circulation (Kolodin et al., 2015; Vasanthakumar et al., 2020), indicating that continual, low-level recruitment of $T_{R}$ to VAT occurs throughout adulthood. Thus, longerterm antibody-mediated blockade may be necessary to observe significant effects on $T_{R}$ accumulation in our system. Additionally, addressing the role of CCR3 is further complicated by its promiscuity, and whether compensation through other ligands occurs during in vivo blockade is unknown. In vitro, we found that ICOS stimulation resulted in reduced CCR3 expression in WT VAT- $T_{R}$ with no changes in YF or KO cultures, suggesting that ICOS-dependent PI3K signaling inhibits expression of CCR3. Control of CCR3 expression in T cells has not been extensively characterized, however there is evidence that the transcription factor GATA3 is able to bind to a regulatory region in the Ccr3 locus (Kong et al., 2013). Nonlymphoid $T_{R}$, including VAT- $T_{R}$, express high levels of GATA3 (Cipolletta et al., 2012; Delacher et al., 2020). However, whether ICOS signaling impacts CCR3 expression by modulating GATA3 expression or activity, and whether GATA3 is involved in controlling CCR3 expression in $T_{R}$, remains to be explored.

Most studies assessing VAT- $T_{R}$ biology utilize older male mice due to an increased abundance of canonical VAT- $T_{R}$ with age (Cipolletta et al., 2015). However, mediators of tissue inflammation, including IL-6, TNFa, and IL1 $\beta$, are upregulated in the VAT of aged male mice (older than 24 weeks) (Wu et al., 2007). We observed consistent accumulation of VAT- $T_{R}$ from 5 weeks on in all three genotypes, which was correlated with an increase in CCR $3^{+} \mathrm{T}_{\mathrm{R}}$ up to $\sim 20$ weeks of age. CCR2 regulates T cell access to sites of inflammation (Lee et al., 2007), and studies assessing the contribution of CCR2 on $T_{R}$ migration to VAT utilize mice aged 25-30 weeks, when measures of VAT inflammation are increasing. CCR2 and CCR3 likely both have important roles for promoting recruitment of $T_{R}$ to VAT, however it will be necessary to evaluate whether 
these receptors are utilized differentially based on age, sex, and inflammation. For example, CCR3 may be one important factor for early seeding and continuous recruitment of $T_{R}$ to VAT in comparatively younger mice when VAT inflammation is low. With increasing age and inflammation (particularly in male mice), a switch to CCL2CCR2 mediated migration may dominate.

Future studies are required to understand how ICOS exerts tissue-specific effects. The skin and lungs are under chronic stimulation by environmental antigens and commensal microbes, whereas the sterile VAT is maintained in a $T_{H} 2$-skewed environment in metabolically healthy mice. The milieu of chemokines and cytokines expressed at baseline in these tissues is likely very different. Indeed, $T_{R}$ express distinct patterns of homing receptors and adhesion molecules to appropriately access specific nonlymphoid tissues (reviewed in Campbell \& Koch, 2011). Absence of ICOS signaling may support expression of chemokine receptors that preferentially drive $T_{R}$ to VAT due to constitutive expression of unique chemokines within this tissue. Additionally, VAT- $T_{R}$ may rely less on PI3K signals compared to $T_{R}$ found from other tissues, due to the presence of other supporting factors in the VAT of naïve mice, such as high levels of IL33. Indeed, a recent study demonstrated that insulin receptor deletion in $T_{R}$, which also results in reduced PI3K signaling, led to an increased suppressive phenotype in VAT- $T_{R}$ and enhanced insulin sensitivity (Wu et al., 2020). PI3K-mediated activation of mTORC1 also promotes glycolytic metabolism in T cells. Thus, the ability of the mTORC1 inhibitor rapamycin to diminish Treg cell abundance in the spleen but not the VAT suggests that Treg cells in different tissue sites may have distinct metabolic requirements for their homeostatic maintenance.

We describe a surprising, antagonistic role for ICOS-dependent PI3K signaling in the accumulation, prototypical phenotype, and function of $T_{R}$ specifically in VAT. We suggest that absence of ICOS signaling supports enhanced recruitment of $T_{R}$ to VAT associated with increased expression of CCR3. Further studies will be needed to identify the molecular mechanisms by which ICOS inhibits the VAT- $T_{R}$ phenotype and CCR3 expression. This highlights the complexity of tissue-specific $T_{R}$ development and accumulation and the importance of considering how signals from the immune environment elicit both cell- and tissue-specific effects. 


\section{Materials and Methods}

\section{Mice}

CD45.1 B6.SJL (B6.SJL-Ptprc ${ }^{a} P e p c^{b} /$ BoyJ), B6.Icos ${ }^{-/-}$(B6.129P2-Icos ${ }^{\text {tm1Mak} / J), ~ a n d ~}$ B6.Foxp $3^{\text {mRFP }}\left(\mathrm{C} 57 \mathrm{BL} / 6-F o x p 3^{\text {tm1F/v}} / \mathrm{J}\right)$ mice were purchased from The Jackson Laboratory. B6. Icos ${ }^{Y 181 F}$ mice were provided by M. Pepper (University of Washington, Seattle, WA). All mice were backcrossed to a C57BL/6J background for at least 10 generations. Foxp $3^{\text {mRFP }}$ mice were used as WT controls and mice from different genotypes used were co-housed at weaning. CD45.1 $1^{+}$B6.SJL mice were crossed to B6. Foxp3 ${ }^{\text {mRFP }}$ mice to generate Foxp3 ${ }^{\text {mRFP }}$ mice expressing CD45.1, CD45.2, and CD45.1/.2 allelic variants. B6. Icos ${ }^{-1-}$ and B6. I $\cos ^{Y 181 F}$ mice were crossed to B6. Foxp $3^{\text {mRFP }}$ mice to generate B6. $\cos ^{-/-}$and B6.Icos ${ }^{Y 181 F}$ mice expressing $F_{O X p} 3^{m R F P}$. All animals were bred and housed in specific pathogen-free conditions under the approval of the Institutional Animal Care and Use Committee of the Benaroya Research Institute._Males were used unless specified otherwise. Age of mice is indicated per experiment.

\section{Mixed bone marrow chimeras}

Bone marrow cells were prepared from donor mice by flushing femurs and tibias with PBS. Red blood cells (RBC) from filtered cells were lysed in ACK lysis buffer (Life Technologies) for 5 min at room temperature (RT). Recipient mice were lethally irradiated ( $2 \times 600$ rad separated by $\geq 4 \mathrm{hr}$ ). Mixed bone marrow chimeras were generated by retro-orbitally (r.o.) injecting a $1: 1$ ratio $\left(\geq 2 \times 10^{6}\right.$ million cells total) of bone marrow cells of the appropriate genotypes into anesthetized recipient mice. Chimeric mice were rested for $\geq 10$ wk.

\section{Intravascular labeling}

Mice were anesthetized with 4\% isoflurane and $3 \mu \mathrm{g} \mathrm{BV510-} \mathrm{or} \mathrm{BV650-conjugated}$ CD45 (30-F11) was injected into mice r.o. in $200 \mu \mathrm{L}$ PBS 5 min prior to sacrifice. Single cell suspensions were prepared for flow cytometry as described below, and localization of cells was determined by positive (blood-exposed) and negative (tissue-restricted) staining.

\section{Cell isolation}

Single-cell suspensions were prepared from spleen and peripheral LNs (pooled inguinal, axillary, brachial, and superficial cervical nodes) by tissue disruption with frosted glass slides into RPMI with 10\% bovine calf serum (R-10, BCS) and filtered through nitex mesh. Blood was collected via cardiac puncture into PBS containing $2 \mathrm{mM}$ EDTA. Epididymal visceral adipose (VAT) and inguinal subcutaneous adipose (SQAT) were excised, finely minced with scissors, and digested in RPMl basal medium with $0.14 \mathrm{U} / \mathrm{mL}$ Liberase TM (Roche) and $10 \mathrm{U} / \mathrm{mL}$ DNase I (Roche) for $30 \mathrm{~min}$ at $37^{\circ} \mathrm{C}$ with shaking (200 rpm). Supernatants were filtered through a $100 \mu \mathrm{M}$ cell strainers and washed several times to remove mature adipocytes from the stromal vascular fraction. Ears were harvested for skin-infiltrating cells. Dorsal and ventral sides were separated using forceps and digested as described for VAT/SQAT for 45 min. Lungs were digested as described for skin. Large intestines were cleaned, stripped of fatty tissue, 
and inverted. Tissue was placed into extraction media (RPMI basal medium, 2 mM DTT, $1 \mathrm{mM}$ EDTA, $2 \% \mathrm{BCS}$ ) and shaken at $37^{\circ} \mathrm{C}$ for $20 \mathrm{~min}$ to release intraepithelial lymphocytes (IEL). IEL-containing supernatant was removed and filtered into $50 \mathrm{~mL}$ conical tubes. For lamina propria lymphocyte (LPL) isolation, immediately following incubation with extraction media, tissue was finely minced and placed into digestion media (RPMI basal media, $300 \mathrm{U} / \mathrm{mL}$ collagenase I [Worthington Biochemical Corporation], $1 \% \mathrm{BCS}$ ), shaken for $30 \mathrm{~min}$ at $37^{\circ} \mathrm{C}$, and filtered into $50 \mathrm{~mL}$ conical tubes. Isolated cells from all tissues were incubated in ACK lysis buffer for $5 \mathrm{~min}$, washed with R-10, and stained for flow cytometry or cultured for intracellular cytokine or chemokine receptor staining.

\section{Flow cytometry \& intracellular cytokine staining}

Single-cell suspensions were stained with Fixable Viability Dye eFluor 780 (eBioscience) in PBS for $10 \mathrm{~min}$ at RT. For surface staining, cells were incubated at $4^{\circ} \mathrm{C}$ for 30 min in FACS buffer (PBS, 2\% BCS) with directly conjugated antibodies (Abs) for murine proteins. Abs purchased from BioLegend unless noted: CD4 (RM4-5), CD8 (536.7), CD11b (M1/70), CD11c (N418), CD25 (PC61), CD44 (IM7), CD45 (30-F11), CD45.1 (A20), CD45.2 (104), CD62L (MEL-14), CD69 (H1.2F3), CD206 (C068C2), CXCR3 (CXCR3-173), F4/80 (BM8), Gr1 (RB6-8C5), ICOS (15F9), KLRG1 (2F1/KLRG1), Siglec-F (E50-2440; BD Biosciences), ST2 (U29-93; BD Biosciences), TCR $\beta$ (H57-597). For intracellular staining, cells were stained for surface antigens as described above, washed, and permeabilized for 20 min with eBioscience Fix/Perm buffer at $4^{\circ} \mathrm{C}$. Cells were washed and stained in PermWash buffer (eBioscience) for 30 min at $4^{\circ} \mathrm{C}$ with Abs (purchased from BioLegend unless noted) against $\mathrm{Bcl} 2$ (BCL/10C4), CTLA-4 (UC10-4B9), Foxp3 (FJK-16s; Invitrogen), IFNy (XMG1.2; BD Biosciences), IL-10 (JES5-16E3), Ki67 (11F6), TNFa (MP6-XT22). For intracellular cytokine staining following restimulation, cells were incubated in FACS tubes with PMA $(50 \mathrm{ng} / \mathrm{mL})$ and ionomycin $(1 \mu \mathrm{g} / \mathrm{mL})$ plus monensin $(2 \mu \mathrm{M})$ in $0.3 \mathrm{~mL}$ complete RPMI medium (RPMI-C) [RPMI with 10\% (vol/vol) fetal bovine serum, $100 \mathrm{U} / \mathrm{mL}$ penicillin, 100 $\mu \mathrm{g} / \mathrm{mL}$ streptomycin, $25 \mu \mathrm{g} / \mathrm{mL}$ gentamycin, $1 \mathrm{mM}$ sodium pyruvate, $10 \mathrm{mM}$ HEPES, 2 $\mathrm{mM}$ L-glutamine, $55 \mu \mathrm{M} \beta-\mathrm{ME}]$ for $4 \mathrm{hr}$ at $37^{\circ} \mathrm{C}, 5 \% \mathrm{CO}_{2}$ prior to staining as described above. Loss of mRFP expression occurred with our fixation/permeabilization protocols, requiring intracellular Foxp3 staining. Data were acquired on LSR II or FACSCanto flow cytometers (BD Biosciences) and analyzed using FlowJo software (TreeStar). Due to intraexperimental variability, geometric MFI (gMFI) was normalized as fold change compared to average expression in WT samples per experiment. Polybead polystyrene nonfluorescent microspheres (15 mm, Polysciences) were used to determine absolute cell numbers in flow cytometry samples. $100 \mu \mathrm{L}$ of a fixed concentration $\left(\mathrm{C}_{\mathrm{B}}\right)$ of beads was mixed with $100 \mu \mathrm{L}$ cells to be counted. Samples were acquired on a flow cytometer, with gates drawn on lymphocyte and bead populations based on their forward- and side-scatter properties. The ratio of lymphocyte gate events $\left(\mathrm{N}_{\mathrm{L}}\right)$ to bead gate events $\left(N_{B}\right)$ was determined and used to calculate the concentration $\left(C_{L}\right)$ of the original cell suspension as follows: $C_{L}=\left(N_{L} / N_{B}\right) \times C_{B}$ 


\section{Phospho-flow cytometry staining}

$\sim 1 / 4$ of each spleen was immediately disrupted between frosted glass slides into BD

Fix/Perm buffer (BD Biosciences). Cells were incubated for 20 min at RT, washed in FACS buffer, and resuspended in $90 \%$ ice cold methanol for $\geq 30$ min. Cells were washed with BD Perm/Wash buffer (BD Biosciences) and stained for surface and intracellular antigens, including p-S6 (D57.2.2E; Cell Signaling Technology) for $45 \mathrm{~min}$ at RT.

\section{Chemokine receptor staining}

Freshly isolated cells were incubated for $2.5 \mathrm{hr}$ at $37^{\circ} \mathrm{C}, 5 \% \mathrm{CO}_{2}$ in RPMI-C. For CCR7 expression, cells were incubated with CCL19-human IgGFc fusion protein for $20 \mathrm{~min}$ at $4^{\circ} \mathrm{C}$, washed, then incubated with PE-conjugated goat anti-human IgGFc (Jackson ImmunoResearch Laboratories) for $20 \mathrm{~min}$ at $4^{\circ} \mathrm{C}$. To detect CCR2 (475301; R\&D Systems) and CCR3 (J073E5; BioLegend), cells were incubated for $30 \mathrm{~min}$ at $37^{\circ} \mathrm{C}$ with directly conjugated antibodies diluted in FACS buffer. Cells were washed twice with FACS buffer before being acquired on the flow cytometer.

\section{Histology}

VAT was excised, immediately fixed in $10 \%$ formalin for $24 \mathrm{hr}$, then paraffin-embedded. Hematoxylin and eosin (H\&E) staining was performed on $6 \mu \mathrm{m}$ tissue sections by the Benaroya Research Institute Histology Core. Slides were imaged at room temperature with a Leica DM E brightfield microscope with a 10X eyepiece and 20X objective lens (together 200X). Images were caputured with a Leica EC3 3.1 megapixel digital color camera and imported into Leica Application Suite EZ imaging software.

\section{RNA extraction and quantitative PCR}

VAT was dissected, immediately stabilized in RNAlater (Thermo Fisher), and frozen at $20^{\circ} \mathrm{C}$ until ready for processing. $100 \mathrm{mg}$ tissue was homogenized in $1 \mathrm{~mL}$ Qiazol and RNA was extracted using RNeasy Lipid Tissue Mini Kit (Qiagen) according to the manufacturer's instructions. RNA quality and quantity was determined using an ND1000 spectrophotometer (NanoDrop, Thermo Fisher). 500 ng template RNA was reverse transcribed with random hexamer primers in $20 \mu \mathrm{L}$ using RevertAid First Strand cDNA Synthesis Kit (Thermo Fisher) and subsequently diluted 1:3.3 with nuclease-free water. QPCR was performed using $2 \mu \mathrm{L}$ diluted $\mathrm{CDNA}$ and presynthesized TaqMan Gene Expression assays in TaqMan Gene Expression Master Mix (Applied Biosystems) for amplification of the following transcripts in a final volume of $20 \mu \mathrm{L}$ : Ccl5 (Mm01302427_m1), Ccl7 (Mm00443113_m1), Ccl11 (Mm0441238_m1), Ccl24 (Mm00444701_m1), and Ccl26 (Mm02763057_u1). Samples were run in technical triplicates using the QuantStudio 5 Real-Time PCR System (Thermo Fisher) with 10 min initial activation at $95^{\circ} \mathrm{C}$ followed by 40 cycles of $15 \mathrm{sec}$ at $95^{\circ} \mathrm{C}, 60 \mathrm{sec}$ at $60^{\circ} \mathrm{C}$. Mean target mRNA levels were calculated by $\Delta \Delta C T$ method and normalized to Tbp (Mm01277042_m1) expression using QuantStudio Design and Analysis Software (Thermo Fisher). 
VAT was pooled from 2-3 mice of the same genotype to ensure adequate cell number for culture. Cells were isolated as described above, and CD4 ${ }^{+} \mathrm{T}$ cells were enriched by incubating cells with CD4 MicroBeads and positively selecting with MACS cell separation MS columns (Miltenyi Biotec). Purified cells were resuspended in RPMI-C with $500 \mathrm{U} / \mathrm{mL}$ recombinant IL-2 (eBioscience). Cells were cultured for $48 \mathrm{hr}$ in 96 -well flat-bottom plates with plate-bound aCD3 (2C11) and aCD28 (37.51) from Bio X Cell at $1 \mu \mathrm{g} / \mu \mathrm{L}$, with or without the addition of plate-bound alCOS (C398.4A; BioLegend) at 2 $\mu \mathrm{g} / \mu \mathrm{L}$. Expression of CCR3 was assessed by flow cytometry as described above.

\section{Rapamycin treatment}

Mice were given rapamycin $(1 \mathrm{mg} / \mathrm{kg})$ three times per week for three weeks by intraperitoneal injection in PBS containing 5.2\% PEG-400, 5.2\% Tween 80, and 0.5\% ethanol. Control animals were given vehicle only. Mice were euthanized for analysis of Treg cell abundance in the spleen and VAT 2 days after the final injection.

In vivo antibody blockade

Mice aged 8-10 weeks were given $0.75 \mu \mathrm{g} / \mathrm{g}$ body weight $\mathrm{aCCL} 11$ and aCCL24 (R\&D Systems; MAB420 and MAB528) or an equivalent amount of rat IgG (Sigma) diluted in PBS by intraperitoneal (i.p.) injection on days 0,5 , and 10 and sacrificed for analysis on day 13.

\section{High-fat diet}

Mice were placed on 18-week HFD (60\% kcal fat diet, Research Diets D124928, ad libitum) beginning at 5-7 weeks of age. Weights and blood glucose (BGL) by tail prick (Contour Next One glucose meter; Ascensia Diabetes Care) were taken weekly after 6 hr of fasting.

Insulin and glucose tolerance tests

Insulin tolerance tests were performed in conscious mice fasted for $6 \mathrm{hr}$. $1 \mathrm{U} / \mathrm{kg}$ human insulin (Sigma) was given i.p. and blood was collected by tail prick for BGL at 0, 15, 30, 45, and 60 min post insulin administration. Three days later, glucose tolerance tests were performed in conscious mice fasted for $6 \mathrm{hr} .1 \mathrm{~g} / \mathrm{kg}$ glucose (Sigma) was administered i.p. Blood was collected by tail prick for BGL at 0, 15, 30, 60, 90, and 120 min post-glucose bolus .

\section{Statistical analysis}

All data are presented as mean values \pm SD and graphs were created and analyzed using Prism Software (GraphPad). Comparisons between genotypes were analyzed using one- or two-way ANOVA where appropriate, adjusted for multiple comparisons using Tukey's post-test. For mixed bone marrow chimeras, statistical significance was determined using two-tailed paired Student's $t$ tests when comparing cells within the same chimeric mouse.

\section{Supplemental material}

Fig. S1 shows ICOS expression across gated splenic $T_{R}$ populations and summarizes $\mathrm{pLN} \mathrm{T}_{\mathrm{R}}$ frequency. Fig. $\mathrm{S} 2$ shows further characterization of splenic and VAT- $\mathrm{T}_{\mathrm{R}}$ by flow 
cytometry. Fig. S3 shows the impact of rapamycin treatment on Treg cell abundance in the spleen and VAT. Fig. S4 shows VAT histology (H\&E staining) and frequencies of ATM and ILC2 populations by flow cytometry. Fig. S5 shows further characterization of mixed bone marrow chimeras. Fig. S6 expands on CCR2 and CCR3 expression in specific tissues in both intact and chimeric mice (flow cytometry), shows expression of CCR3 ligands in whole VAT (qPCR), and characterizes eosinophil frequencies in VAT after in vivo CCL11/24 blockade

\section{Figure Legends}

Figure 1: Absence of ICOS signaling results in loss of lymphoid $T_{R}$ and altered expression of PI3K targets. (A) Representative flow cytometry plots and quantification of splenic $\mathrm{T}_{\mathrm{R}}\left(n=3-6\right.$ per group from 9 independent experiments). (B) Splenic $\mathrm{T}_{\mathrm{R}}$ frequencies at indicated ages ( $n=3-5$ per group from 16 independent experiments). (C) Representative flow cytometry plots and quantification of splenic $\mathrm{CT}_{\mathrm{R}}\left(\mathrm{CD} 44^{\mathrm{lo}} \mathrm{CD} 62 \mathrm{~L}^{\mathrm{hi}}\right)$ and $\mathrm{eT}_{\mathrm{R}}\left(\mathrm{CD} 44^{\text {hi }} \mathrm{CD} \mathrm{L}^{\mathrm{l}}{ }^{\circ}\right.$ ) ( $n=3-5$ per group from 7 independent experiments). (D) Expression of CD62L and CCR7 in splenic $\mathrm{T}_{\mathrm{R}}$ (CD62L: $n=3-5$ per group from 5 independent experiments. CCR7: $n=3-5$ per group from 2 independent experiments). (E) S6 phosphorylation measured directly ex vivo in splenic $T_{R}$ by flow cytometry. ( $n=$ 3-4 per group from 2 independent experiments). Mice were age-matched within independent experiments and pooled data are from experiments using mice aged 8-16 weeks, except in (B) where age is indicated. Statistical significance was determined using one-way ANOVA with Tukey's post-test. All graphical data are presented as mean values \pm SD.

Figure 2: $T_{R}$ frequency and number are increased in VAT in the absence of ICOS signaling. (A-C) Representative flow cytometry plots and quantification of of $T_{R}$ among tissue-localized TCR $\beta^{+} C D 4^{+}$cells in WT, YF, and KO ear skin (A), lung (B), VAT (C), and inguinal SQAT (D) of male mice (Skin: $n=3$ per group from 2 independent experiments. Lung: $n=3$ per group from 3 independent experiments. VAT: $n=3-6$ from 8 independent experiments. SQAT: $n=3-5$ for 5 independent experiments). (E) Tissuelocalized VAT- $T_{\mathrm{R}}$ frequency and total number in female WT, YF, and KO mice $(n=3$ per group for 3 independent experiments). (F) $T_{R}$ frequency and total number in VAT of male versus female WT mice (combined data from WT groups in (C) and (E)). (G) VAT$\mathrm{T}_{\mathrm{R}}$ frequencies by noted age bins in male mice as measured by flow cytometry $(n=3-5$ mice per group from 18 independent experiments). Mice were age-matched within independent experiments and pooled data are from experiments using mice aged 8-16 weeks, except in (G) where age is indicated. Statistical significance was determined using one-way ANOVA with Tukey's post-test or two-tailed Student's $t$ test where appropriate. All data are presented as mean values \pm SD.

Figure 3: Loss of ICOS signaling supports an $\mathrm{eT}_{\mathrm{R}}$ phenotype in VAT. (A) Representative flow cytometry plots and quantification of KLRG1, CD69, CTLA-4, and CD25 expression in tissue-localized VAT- $T_{R}$. CTLA-4 gMFI was calculated as fold change compared to average expression in WT $\mathrm{T}_{\mathrm{R}}$ for each individual experiment $(n=$ 3-6 per group from 3 independent experiments or for CTLA-4, 2 independent 
experiments). (B) Representative gating of VAT- $\mathrm{T}_{\mathrm{R}}$ on CXCR3 and ST2 expression and quantification of VAT- $T_{\mathrm{R}}$ positive for CXCR3 (left) or ST2 (right) alone, or doubleexpressers of CXCR3 and ST2 (middle) ( $n=3-5$ per group from 5 independent experiments). (C) Representative histograms showing expression of KLRG1 and CD69 in $\mathrm{CXCR}^{+} \mathrm{ST} 2^{+} \mathrm{YF}$ and KO VAT-T $\mathrm{R}$ as measured by flow cytometry. (D) Representative flow cytometry plots and quantification of IL-10 expression in $T_{R}$ from VAT and spleen after 4h PMA/I+monensin stimulation ex vivo ( $n=3-5$ per group from 2 independent experiments). Mice were age-matched within independent experiments and pooled data are from experiments using male mice aged 8-16 weeks. Statistical significance was determined using one-way ANOVA with Tukey's post-test. All data are presented as mean values $\pm S D$.

Figure 4: Reduced expression of inflammatory markers in YF and KO VAT. (A) Summary of total mouse and VAT weight in male WT, YF and KO mice $(n=3-6$ per group from 6 independent experiments). (B) Representative flow cytometry plots and quantification of tissue-localized VAT- eff $_{\text {in }} \mathrm{WT}$, YF, and KO mice $(n=3-6$ per group from 8 independent experiments). (C) Representative gating of VAT-T eff $_{\text {On }}$ CXCR3 and

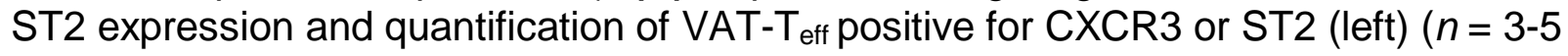
per group from 5 independent experiments). Representative flow cytometry plots (top panels) and quantification (bottom panels) of IFNy, TNFa, and IL-10 expression in VAT$\mathrm{T}_{\text {eff }}$ after $4 \mathrm{~h} \mathrm{PMA} / \mathrm{I}+$ monensin stimulation ex vivo (right) $(n=3-5$ per group from 2 independent experiments). (D) Tissue-localized eosinophil frequencies in VAT ( $n=2-6$ from 6 independent experiments). Mice were age-matched within independent experiments and pooled data are from experiments using male mice aged 8-16 weeks. Statistical significance was determined using one-way ANOVA with Tukey's post-test. All graphical data are presented as mean values \pm SD.

Figure 5: Mice deficient in ICOS signaling maintain anti-inflammatory immune cell abundance and phenotype in VAT after long-term HFD, correlating with improved insulin sensitivity. (A) Percentage of baseline body weight at indicated timepoints following initiation of HFD. (B) Tissue-localized VAT- $T_{R}$ frequency after 18 weeks of HFD. (C) VAT- $T_{R}$ phenotypic data as measured by flow cytometry showing expression of CXCR3, ST2, and KLRG1. (D) Frequency of tissue-localized eosinophils in VAT. (E) Representative gating of VAT- $\mathrm{T}_{\text {eff }}$ on CXCR3 and ST2 with summarized graphical data. (F) Fasting BGL (top) and GTT (bottom) in HFD-fed mice. (G) ITT in HFD-fed mice. * indicates significant difference between WT and YF; \# indicates significant difference between WT and KO. ITT area under the curve (AUC) was calculated for each individual mouse. All data are representative of 2 independent experiments with $n=4-5$ per group. For weight (A), GTT, and ITT (F, G), statistical significance was determined using two-way repeated measures ANOVA with Tukey's post-test for multiple comparisons. For fasted BGL (F), ITT AUC (G), and all flow cytometry summary graphs, statistical significance was determined using one-way ANOVA with Tukey's post-test. All graphs are presented as mean values \pm SD.

Figure 6: Cell-intrinsic ICOS signaling limits $T_{R}$ and $T_{\text {eff }}$ abundance and phenotype in VAT. (A) Schematic of mixed bone marrow chimera set-up (left) and analysis (right). 
(B) Representative gating of CD45.1 vs. CD45.2 expression in chimeric mice on tissuelocalized $T_{R}$ in VAT and quantification of donor $T_{R}$ reconstitution in VAT. (C) Representative gating on CXCR3- and ST2-expressing CD45.1 $1^{+}$and CD45.2 $2^{+}$donor VAT- $T_{R}$ in chimeric mice (top). Lines connect points indicating CD45.1 $1^{+}$and $C D 45.2^{+}$ cells within the same chimeric mouse. Histogram and quantification of CD69 expression in donor VAT- $T_{R}$. Histogram and graphical summary of donor VAT- $T_{R}$ expression of IL-

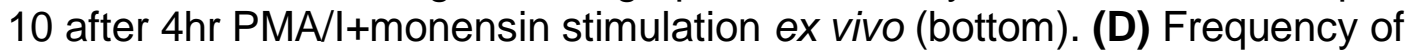
endogenous CD45.1/2 ${ }^{+}$VAT- $T_{R}$ in chimeric mice. (E) Reconstitution of donor $T_{R}$ in the indicated tissues. (F) Summary of donor $T_{\text {eff }}$ reconstitution in the indicated tissues. (G) CXCR3 and ST2 expression by gated VAT- $\mathrm{T}_{\text {eff }}$ (top). Histograms and summary of donor VAT- $T_{\text {eff }}$ expression of IFNy, TNFa, and IL-10 after $4 \mathrm{hr}$ PMA/I+monensin stimulation ex vivo (bottom). Data are pooled from 3 independent experiments with $n=3-5$ per group. Statistical significance for cell population reconstitution was determined using one-way ANOVA with Tukey's post-test for each tissue analyzed. Data are presented as mean values $\pm S D$. A two-tailed, paired Student's $t$ test was used to assess statistical significance when comparing expression in donor cells within the same chimeric mouse.

\section{Figure 7: Increased abundance of $Y F$ and KO VAT- $T_{R}$ is not due to enhanced proliferation or Bcl2 expression. (A, B) Ki67 (A) and Bcl2 expression (B) in tissue- localized VAT- $T_{R}$ as measured by flow cytometry directly ex vivo. Bcl2 gMFI was} calculated as fold change compared to average expression in WT $T_{R}$ for each individual experiment (Ki67: $n=3-5$ per group from 3 independent experiments. Bcl-2: $n=3-5$ per group from 2 independent experiments). Mice were age-matched within independent experiments and pooled data are from experiments using male mice aged 8-16 weeks. Statistical significance was determined using one-way ANOVA with Tukey's post-test. All data are presented as mean values \pm SD.

Figure 8: Increased VAT- $T_{R}$ accumulation in the absence of ICOS signaling is associated with elevated CCR3 expression. (A) ST2 expression in splenic $T_{R}(n=3$ 5 per group from 8 independent experiments). (B) ST2 expression in CD45.1 $1^{+}$and CD45.2 $2^{+}$donor splenic $T_{R}$ in WT:YF and WT:KO chimeric mice. Lines connect CD45.1 $1^{+}$ and $\mathrm{CD} 45.2^{+}$donor splenic $\mathrm{T}_{\mathrm{R}}$ within the same chimera $(n=3-5$ chimeric mice per group from 3 independent experiments). (C) Expression of CCR3 in VAT- $T_{R}$ in mice $\leq 8$ wk and $>8$ wk of age ( $n=3-5$ per group from 7 independent experiments). (D) Expression of CCR2 and CCR3 by VAT- $\mathrm{T}_{\mathrm{R}}(n=3-5$ per group from 2 independent experiments). (E) Expression of indicated CCR3 ligands in total VAT normalized to Tbp as measured by qPCR ( $n=5$ per group). (F) Schematic of in vitro culture experiments examining the impact of ICOS signaling on CCR3 expression (left). Graphs indicating fold change in $\mathrm{T}_{\mathrm{R}}$ frequency of $\mathrm{CD} 4^{+}$cells and $\% \mathrm{CCR}^{+}$of $\mathrm{T}_{\mathrm{R}}$ between individual culture samples stimulated with or without alCOS for $2 \mathrm{~d}$ (middle). Representative flow cytometry plots with frequency of $C C R 3^{+} T_{R}$ after $2 d$ in specified culture conditions (right) ( $n=1-3$ per group from 3 independent experiments). (G) (Left) Representative flow cytometry plots indicating VAT- $T_{R}$ frequency with or without CCL11/24 blockade. Graphs summarize $\mathrm{T}_{\mathrm{R}}$ frequencies in indicated tissues after $2 \mathrm{wk}$ ( $n=3-4$ per group). Mice were age-matched within independent experiments and collectively, pooled data are from experiments using male mice aged 8-16 weeks unless otherwise indicated. 
Statistical significance was determined using one-way ANOVA with Tukey's post-test (A, C), two-tailed, paired Student's $t$ test for expression in donor cells within the same chimeric mouse (B), and two-tailed Student's $t$ test $(\mathbf{F}, \mathbf{G})$. All data are presented as mean values $\pm S D$.

Supplemental Figure 1 (goes with Figure 1): Despite normal surface expression of ICOS, YF mice phenocopy KO with equivalent loss of lymphoid $T_{R}$. (A) ICOS expression on gated WT, YF, and KO splenic $T_{R}$. Fold change of ICOS gMFI is compared to average expression in WT $\mathrm{T}_{\mathrm{R}}$ for each individual experiment $(n=3$ per group from 5 independent experiments). (B) Representative ICOS expression on gated splenic $\mathrm{KO}$ and $\mathrm{WT} C D 44^{\mathrm{lo}} \mathrm{CD} 62 \mathrm{~L}^{\mathrm{hi}} \mathrm{CT}_{\mathrm{R}}$ and $\mathrm{CD} 44^{\mathrm{hi}} \mathrm{CD} 62 \mathrm{~L}^{\mathrm{lo}} \mathrm{eT}_{\mathrm{R}}$ as measured by flow cytometry ( $n=3$ per group from 5 independent experiments). (C) $\mathrm{T}_{\mathrm{R}}$ frequencies of total CD4 ${ }^{+} \mathrm{T}$ cells in pLN ( $n=2-5$ per group from 5 independent experiments). Mice were age-matched within individual experiments and quantified data are pooled from experiments using male mice 8-16 weeks of age. Statistical significance was determined using one-way ANOVA with Tukey's post-test. All data are presented as mean values $\pm S D$.

Supplemental Figure 2 (goes with Figure 3): Expression of activation markers on splenic and VAT-T R. $_{\text {. }}$ (A) KLRG1 and CD69 expression in gated WT, YF, and KO splenic $\mathrm{T}_{\mathrm{R}}$ ( $n=3-6$ per group from 3 independent experiments). (B) CD44 and Foxp3 expression in tissue-localized VAT- $T_{R}$. gMFI was calculated as fold change compared to average expression in WT $\mathrm{T}_{\mathrm{R}}$ for each individual experiment $(n=3-6$ per group from 6 independent experiments). Mice were age-matched within individual experiments and pooled data are from experiments using 8-16 week old male mice. Statistical significance was determined using one-way ANOVA with Tukey's post-test. All data are presented as mean values \pm SD.

Supplemental Figure 3 (goes with Figure 3): Rapamycin treatment reduces Treg cell numbers in spleen but not VAT. Number of $\mathrm{CD}^{+}{ }^{+} \mathrm{Foxp} 3^{+} \mathrm{T}$ reg cells in spleen (left) and VAT (right) in mice treated with rapamycin $(1 \mathrm{mg} / \mathrm{kg})$ or vehicle as indicated ( $\mathrm{n}=5$ mice per group from one experiment). Statistical significance was determined using two-tailed unpaired Student's $t$ test. All data are presented as mean values \pm SD.

Supplemental Figure 4 (goes with Figure 4): Adipocyte, ATM, and ILC2 populations are unchanged in YF and KO VAT. (A) H\&E staining of VAT from 8 week old male mice. (B) Representative flow cytometry plots and quantification of tissuelocalized ATM in WT, YF, and KO mice (top). Representative gating of ATM on CD11c and CD206 to identify M1-like and M2-like macrophages, respectively (bottom) ( $n=2-6$ per group from 6 independent experiments). (C) Frequency of tissue-localized ILC2s in VAT as measured by flow cytometry $(n=2-6$ per group from 6 independent experiments). Mice were age-matched within individual experiments and pooled data are from experiments using 8-16 week old male mice. Statistical significance was determined using one-way ANOVA with Tukey's post-test. All data are presented as mean values \pm SD. 
Supplemental Figure 5 (goes with Figure 6): Cell- and tissue-specific changes in reconstitution in ICOS mutant mixed bone marrow chimeras. (A) Summary of total mouse (left), VAT (middle), and SQAT (right) weights in mixed bone marrow chimeras. (B) Representative gating of CD45.1 vs. CD45.2 expression on tissue-restricted $T_{R}$ in intraepithelial lymphocytes (IEL) from the large intestine. Graphs summarize reconstitution of donor $T_{R}$ of IEL and lamina propria lymphocytes (LPL) in the LI. (C) Representative gating of CD45.1 vs. CD45.2 expression in chimeric mice on total CD8 ${ }^{+}$ T cells (top) and ILC2s (bottom) in VAT. Graphs summarize reconstitution of donor $\mathrm{CD}^{+} \mathrm{T}_{\text {cells }}$ (top) and ILC2s (bottom) in VAT. Data are pooled from 3 independent mixed bone marrow chimera experiments with $n=3-5$ male chimeric mice per group. Statistical significance was determined using one-way ANOVA with Tukey's post-test for each individual tissue. All data are presented as mean values \pm SD.

Supplemental Figure 6 (goes with Figure 8): Increased accumulation of $\mathrm{CCR}^{+} \mathrm{T}_{\mathrm{R}}$ in the absence of ICOS signaling is specific to VAT. (A) CCR2 expression by splenic (top) and VAT- $\mathrm{T}_{\mathrm{R}}$ (bottom) as measured by flow cytometry $(n=3-5$ per group from 2 independent experiments). (B) Expression of CCR3 in splenic $T_{R}$ in mice $\leq 8$ wk and $>8$ wk of age ( $n=3-5$ per group from 7 independent experiments). (C) Expression of CCR3 by gated CD45.1 $1^{+}$and $\mathrm{CD} 45.2^{+}$donor VAT- $\mathrm{T}_{\mathrm{R}}$ in chimeric mice. Graphs summarize CCR3 expression by donor $T_{R}$ in VAT and spleen. Line connects point representing CD 45. $1^{+}$and CD45. $2^{+}$cells within the same chimeric mouse $(n=2-4$ per group from 2 independent experiments). (D) CCR3 expression by tissue-localized skin $T_{R}$ as measured by flow cytometry ( $n=2-4$ per group from 2 independent experiments). (E) CCR3 expression by CD45.1 $1^{+}$and CD45.2 $2^{+}$donor $T_{R}$ within the same chimeric mouse in indicated tissues. Line connects $\mathrm{CD} 45.1^{+}$and $\mathrm{CD} 45.2^{+}$cells within the same chimeric mouse ( $n=2-4$ per group from 2 independent experiments). (F) Frequency of tissuerestricted VAT-eosinophils with and without in vivo CCL11/24 blockade as measured by flow cytometry ( $n=3-4$ mice per group). Mice were age-matched within individual experiments and pooled data are from experiments using 8-16 week old male mice. Statistical significance was determined using one-way ANOVA with Tukey's post-test (A, B, D), two-tailed, paired Student's $t$ test for expression in donor cells within the same chimeric mouse (C, E), and two-tailed Student's $t$ test (F). All data are presented as mean values $\pm S D$. 


\section{Acknowledgements}

We thank A. Wojno, K. Arumuganathan, and T. Nguyen for maintaining the BRI Flow Cytometry Core; P. Johnson in the BRI Histology/lmaging Core; and D. Hackney and S. Zraika from the UW Diabetes Research Center Cell Function Analysis Core for help with metabolic profiling tests. M. Pepper generously provided $I \cos ^{Y 181 F}$ mice with kind permission from W.-K. Suh. A. Burich, C. Toledano, and the BRI vivarium staff helped maintain mouse colonies. We thank members of the Campbell lab for helpful discussion and laboratory support.

This work was supported by grants to D.J.C. from the NIH (R01Al124693, R01Al136475). K.L.M. was supported by NIH NIAID T32 Grant Al106677, NIGMS T32 Grant GM007270, and the UW Diabetes Research Center Samuel and Althea Stroum Endowed Graduate Fellowship (2P30 DK17047).

Author Contributions: K.L.M. and D.J.C. conceptualized study and designed experiments. K.L.M. performed experiments, analyzed data, and wrote original draft. D.J.C. reviewed and edited.

Disclosures: The authors declare no competing financial interests. 


\section{References}

Ahnstedt, H., M. Roy-O’Reilly, M. S. Spychala, A. S. Mobley, J. Bravo-Alegria, A. Chauhan, J. Aronowski, S. P. Marrelli, and L. D. McCullough. 2018. Sex differences in adipose tissue CD8+ T cells and regulatory T cells in middle-aged mice. Front. Immunol. 9:659. doi:10.3389/fimmu.2018.00659

Ali, N., B. Zirak, R. Rodriguez, M. Pauli, H.-A. Truong, K. Lai, R. Ahn, K. Corbin, M. Lowe, T. Scharschmidt, K. Taravati, M. Tan, R. Ricardo-Gonzalez, A. Nosbaum, M. Bertolini, W. Liao, F. Nestle, R. Paus, G. Cotsarelis, A. Abbas, and M. Rosenblum. 2017. Regulatory T cells in skin facilitate epithelial stem cell differentiation. Cell. 169:1119-1129. doi:10.1016/j.cell.2017.05.002

Anderson, K., K. Mayer-Barber, H. Sung, L. Beura, B. James, J. Taylor, L. Qunaj, T. Griffith, V. Vezys, D. Barber, and D. Masopust. 2014. Intravascular staining for discrimination of vascular and tissue leukocytes. Nat. Protoc. 9:209-222.

doi:10.1038/nprot.2014.005

Arimura, Y., H. Kato, U. Dianzani, T. Okamoto, S. Kamekura, D. Buonfiglio, T. MiyoshiAkiyama, T. Uchiyama, and J. Yagi. 2002. A co-stimulatory molecule on activated T cells, $\mathrm{H} 4 / \mathrm{ICOS}$, delivers specific signals in $\mathrm{T}(\mathrm{h})$ cells and regulates their responses. Int. Immunol. 14:555-566. doi:10.1093/intimm/dxf022

Arpaia, N., J. A. Green, B. Moltedo, A. Arvey, S. Hemmers, S. Yuan, P. M. Treuting, and A. Y. Rudensky. 2015. A distinct function of regulatory T cells in tissue protection. Cell. 162:1078-1089. doi:10.1016/j.cell.2015.08.021

Burhans, M. S., D. K. Hagman, J. N. Kuzma, K. A. Schmidt, and M. Kratz. 2018. Contribution of adipose tissue inflammation to the development of type 2 diabetes mellitus. Compr. Physiol. 9:1-58. doi:10.1002/cphy.c170040

Burmeister, Y., T. Lischke, A. C. Dahler, H. W. Mages, K.-P. P. Lam, A. J. Coyle, R. A. Kroczek, and A. Hutloff. 2008. ICOS controls the pool size of effector-memory and regulatory T cells. J. Immunol. 180:774-782. doi:10.4049/jimmunol.180.2.774

Burzyn, D., W. Kuswanto, D. Kolodin, J. L. Shadrach, M. Cerletti, Y. Jang, E. Sefik, T. G. Tan, A. J. Wagers, C. Benoist, and D. Mathis. 2013. A special population of regulatory T cells potentiates muscle repair. Cell. 155:1282-1295.

doi:10.1016/j.cell.2013.10.054

Busse, M., M. Krech, A. Meyer-Bahlburg, C. Hennig, and G. Hansen. 2012. ICOS mediates the generation and function of CD4+CD25+Foxp3+ regulatory $T$ cells conveying respiratory tolerance. J. Immunol. 189:1975-1982.

doi:10.4049/jimmunol.1103581 
Campbell, D. J. 2015. Control of regulatory T cell migration, function, and homeostasis. J. Immunol. 195:2507-2513. doi:10.4049/jimmunol.1500801

Campbell, D. J. and M. A. Koch. Phenotypical and functional specialization of FOXP3+ regulatory T cells . Nat. Rev. Immunol. 11:119-130. doi:10.1038/nri2916

Cipolletta, D. M. Feuerer, A. Li, N. Kamei, J. Lee, S. E. Shoelson, C. Benoist, and D. Mathis. 2012. PPAR-y is a major driver of the accumulation and phenotype of adipose tissue Treg cells. Nature. 486:549-553. doi:10.1038/nature11132

Cipolletta, D. P. Cohen, B. M. Spiegelman, C. Benoist, and D. Mathis. 2015. Appearance and disappearance of the mRNA signature characteristic of Treg cells in visceral adipose tissue: age, diet, and PPARy effects. Proc. Natl. Acad. Sci. U.S.A. 112:482-487. doi:10.1073/pnas. 1423486112

Cretney, E., A. Xin, W. Shi, M. Minnich, F. Masson, M. Miasari, G. T. Belz, G. K. Smyth, M. Busslinger, S. L. Nutt, and A. Kallies. 2011. The transcription factors Blimp-1 and IRF4 jointly control the differentiation and function of effector regulatory T cells. Nat. Immunol. 12:304-311. doi:10.1038/ni.2006

Danilova, E., I. Skrindo, E. Gran, B. J. Hales, W. A. Smith, J. Jahnsen, F. E. Johansen, F. L. Jahnsen, and E. S. Baekkevold. 2015. A role for CCL28-CCR3 in T-cell homing to the human upper airway mucosa. Nat. Mucosal Immunol. 8:107-114.

doi:10.1038/mi.2014.46

Deiuliis, J., Z. Shah, N. Shah, B. Needleman, D. Mikami, V. Narula, K. Perry, J. Hazey, T. Kampfrath, M. Kollengode, Q. Sun, A. R. Satoskar, C. Lumeng, S. Moffatt-Bruce, and S. Rajagopalan. 2011. Visceral adipose inflammation in obesity is associated with critical alterations in Tregulatory cell numbers. PLoS One. 6:e16376.

doi:10.1371/journal.pone.0016376

Delacher, M., C. Imbusch, D. Weichenhan, A. Breiling, A. Hotz-Wagenblatt, U. Träger, A.-C. Hofer, D. Kägebein, Q. Wang, F. Frauhammer, J.-P. Mallm, K. Bauer, C. Herrmann, P. Lang, B. Brors, C. Plass, and M. Feuerer. 2017. Genome-wide DNAmethylation landscape defines specialization of regulatory $T$ cells in tissues. Nat. Immunol. 18:1160-1172. doi:10.1038/ni.3799

Delacher, M., C. Imbusch, A. Hotz-Wagenblatt, J.-P. Mallm, K. Bauer, M. Simon, D. Riegel, A. Rendeiro, S. Bittner, L. Sanderink, A. Pant, L. Schmidleithner, K. Braband, B. Echtenachter, A. Fischer, V. Giunchiglia, P. Hoffmann, M. Edinger, C. Bock, M. Rehli, B. Brors, C. Schmidl, and M. Feuerer. 2020. Precursors for nonlymphoid-tissue Treg cells reside in secondary lymphoid organs and are programmed by the transcription factor BATF. Immunity. 52:1-18. doi:10.1016/j.immuni.2019.12.002 
DiSpirito, J. R., D. Zemmour, D. Ramanan, J. Cho, R. Zilionis, A. M. Klein, C. Benoist, and D. Mathis. 2018. Molecular diversification of regulatory T cells in non-lymphoid tissues. Sci. Immunol. 3:eaat5861. doi:10.1126/sciimmunol.aat5861

Dominguez-Villar, M., and D. A. Hafler. 2018. Regulatory T cells in autoimmune disease. Nat. Immunol. 19:665-675. doi:10.1038/s41590-018-0120-4

Dong, C., A. E. Juedes, U. A. Temann, S. Shresta, J. P. Allison, N. H. Ruddle, and R. A. Flavell. 2001. ICOS co-stimulatory receptor is essential for T-cell activation and function. Nature. 409:97-101. doi:10.1038/35051100

Eller, K., A. Kirsch, A. M. Wolf, S. Sopper, A. Tagwerker, U. Stanzl, D. Wolf, W. Patsch, A. R. Rosenkranz, and P. Eller. 2011. Potential role of regulatory T cells in reversing obesity-linked insulin resistance and diabetic nephropathy. Diabetes. 60:2954-2962. doi:10.2337/db11-0358

Feito, M., R. Vaschetto, G. Criado, A. Sánchez, A. Chiocchetti, A. Jiménez-Periáñez, U. Dianzani, P. Portoles, and J. Rojo. 2003. Mechanisms of H4/ICOS costimulation: effects on proximal TCR signals and MAP kinase pathways. Eur. J. Immunol. 33:204-214. doi: 10.1002/immu.200390023

Feuerer, M., L. Herrero, D. Cipolletta, A. Naaz, J. Wong, A. Nayer, J. Lee, A. Goldfine, C. Benoist, S. Shoelson, and D. Mathis. 2009. Lean, but not obese, fat is enriched for a unique population of regulatory T cells that affect metabolic parameters. Nat. Med. 15:930-939. doi:10.1038/nm.2002

Fos, C., A. Salles, V. Lang, F. Carrette, S. Audebert, S. Pastor, M. Ghiotto, D. Olive, G. Bismuth, and J. A. Nunès. 2008. ICOS ligation recruits the p50alpha PI3K regulatory subunit to the immunological synapse. J. Immunol. 181:1969-1977.

doi:10.4049/jimmunol.181.3.1969

Francis, J. N., C. M. Lloyd, I. Sabroe, S. R. Durham, and S. J. Till. 2007. T lymphocytes expressing CCR3 are increased in allergic rhinitis compared with non-allergic controls and following allergen immunotherapy. Allergy. 62:59-65. doi:10.1111/j.1398-

9995.2006.01253.x.

Gigoux, M., J. Shang, Y. Pak, M. Xu, J. Choe, T. W. Mak, and W.-K. K. Suh. 2009. Inducible costimulator promotes helper T-cell differentiation through phosphoinositide 3kinase. Proc. Natl. Acad. Sci. U.S.A. 106:20371-20376. doi:10.1073/pnas.0911573106

Guo, L., G. Wei, J. Zhu, W. Liao, W. J. Leonard, K. Zhao, and W. Paul. 2009 IL-1 family members and STAT activators induce cytokine production by Th2, Th17, and Th1 cells. Proc. Natl. Acad. Sci. U.S.A. 106:13463-13468. doi:10.1073/pnas.0906988106

Han, J.M., D. Wu, H. C. Denroche, Y. Yao, C. B. Verchere, and M. K. Levings. 2015. IL33 reverses an obesity-induced deficit in visceral adipose tissue ST2+ T regulatory cells 
and ameliorates adipose tissue inflammation and insulin resistance. $\mathrm{J}$. Immunol. 194:4777-4783. doi:10.4049/jimmunol.1500020

Herman, A., G. Freeman, D. Mathis, and C. Benoist. 2004. CD4+CD25+ T regulatory cells dependent on ICOS promote regulation of effector cells in the prediabetic lesion. J. Exp. Med. 199:1479-1489. doi:10.1084/jem.20040179

Humbles, A. A., B. Lu, D. S. Friend, S. Okinaga, J. Lora, A. Algarawi, T. R. Martin, N. P. Gerard, and C. Gerard. 2002. The murine CCR3 receptor regulates both the role of eosinophils and mast cells in allergen-induced airway inflammation and hyperresponsiveness. Proc. Natl. Acad. Sci. U.S.A. 99:1479-1484. doi:10.1073/pnas.261462598

Hutloff, A., A. Dittrich, K. Beier, B. Eljaschewitsch, R. Kraft, I. Anagnostopoulos, and R. Kroczek. 1999. ICOS is an inducible T-cell co-stimulator structurally and functionally related to CD28. Nature. 397:263-266. doi:10.1038/16717

Ilan, Y., R. Maron, A.-M. Tukpah, T. Maioli, G. Murugaiyan, K. Yang, H. Wu, and H. Weiner. 2010. Induction of regulatory T cells decreases adipose inflammation and alleviates insulin resistance in ob/ob mice. Proc. Nat. Acad. Sci. 107:9765-9770. doi:10.1073/pnas.0908771107

Kalekar, L. A., J. N. Cohen, N. Prevel, P. M. Sandoval, A. N. Mathur, J. M. Moreau, M. M. Lowe, A. Nosbaum, P. J. Wolters, A. Haemel, F. Boin, and M. D. Rosenblum. 2019. Regulatory $T$ cells in skin are uniquely poised to suppress profibrotic immune responses. Sci. Immunol. 4:eaaw2910. doi:10.1126/sciimmunol.aaw2910

Kerdiles, Y. M., D. R. Beisner, R. Tinoco, A. S. Dejean, D. H. Castrillon, R. A. DePinho, and S. M. Hedrick. 2009. Foxo1 links homing and survival of naive T cells by regulating L-selectin, CCR7 and interleukin 7 receptor. Nat. Immunol. 10:176-184.

doi:10.1038/ni.1689

Kohyama, M., D. Sugahara, S. Sugiyama, H. Yagita, K. Okumura, and N. Hozumi. 2004. Inducible costimulator-dependent IL-10 production by regulatory $T$ cells specific for self-antigen. Proc. Natl. Acad. Sci. U.S.A. 101:4192-4197.

doi:10.1073/pnas.0400214101

Kolodin, D., N. van Panhuys, C. Li, A. M. Magnuson, D. Cipolletta, C. M. Miller, A. Wagers, R. N. Germain, C. Benoist, and D. Mathis. 2015. Antigen- and cytokine-driven accumulation of regulatory T cells in visceral adipose tissue of lean mice. Cell. Metab. 21:543-557. doi:10.1016/j.cmet.2015.03.005

Komatsu N., and S. Hori. 2007. Full restoration of peripheral Foxp3+ regulatory T cell pool by radioresistant host cells in scurfy bone marrow chimeras. Proc. Natl. Acad. Sci. U.S.A. 104:8959-8964. doi:10.1073/pnas.0702004104 
Kong, S.-K. K., B. S. Kim, T. G. Uhm, W. Lee, G. R. Lee, C.-S. S. Park, C.-H. H. Lee, and I. Y. Chung. 2013. Different GATA factors dictate CCR3 transcription in allergic inflammatory cells in a cell type-specific manner. J. Immunol. 190:5747-5756. doi:10.4049/jimmunol.1203542

Kornete, M., E. Sgouroudis, and C. A. Piccirillo. 2012. ICOS-dependent homeostasis and function of Foxp3+ regulatory T cells in islets of nonobese diabetic mice. J. Immunol. 188:1064-1074. doi:10.4049/jimmunol.1101303

Landuyt, A., B. Klocke, T. Colvin, T. Schoeb, and C. Maynard. 2019. Cutting Edge: ICOS-deficient regulatory T cells display normal induction of 1110 but readily downregulate expression of Foxp3. J. Immunol. 202:1039-1044. doi:10.4049/jimmunol.1801266

Lee, J. H., S. G. Kang, and C. H. Kim. 2007. FoxP3+ T cells undergo conventional first switch to lymphoid tissue homing receptors in thymus but accelerated second switch to nonlymphoid tissue homing receptors in secondary lymphoid tissues. J. Immunol. 178:301-311. doi:10.4049/jimmunol.178.1.301

Levine, A. G., A. Arvey, W. Jin, and A. Y. Rudensky. 2014. Continuous requirement for the TCR in regulatory T cell function. Nat. Immunol. 15:1070-1078. doi:10.1038/ni.3004

Li, C., J. R. DiSpirito, D. Zemmour, R. G. Spallanzani, W. Kuswanto, C. Benoist, and D. Mathis. 2018. TCR transgenic mice reveal stepwise, multi-site acquisition of the distinctive fat-Treg phenotype. Cell. 174:285-299. doi:10.1016/j.cell.2018.05.004

Luo, C. T., W. Liao, S. Dadi, A. Toure, and M. O. Li. 2016. Graded Foxo1 activity in Treg cells differentiates tumour immunity from spontaneous autoimmunity. Nature. 529:532-536. doi:10.1038/nature16486

Ma, W., P. J. Bryce, A. A. Humbles, D. Laouini, A. Yalcindag, H. Alenius, D. S. Friend, H. C. Oettgen, C. Gerard, and R. S. Geha. 2002. CCR3 is essential for skin eosinophilia and airway hyperresponsiveness in a murine model of allergic skin inflammation. J. Clin. Invest. 109:621-628. doi:10.1172/JCl14097

Matta, B. M., J. M. Lott, L. R. Mathews, Q. Liu, B. R. Rosborough, B. R. Blazar, and H. R. Turnquist. 2014. IL-33 is an unconventional alarmin that stimulates IL-2 secretion by dendritic cells to selectively expand IL-33R/ST2+ regulatory T cells. J. Immunol. 193:4010-4020. doi:10.4049/jimmunol.1400481

Miyamoto, K., C. Kingsley, X. Zhang, C. Jabs, L. Izikson, R. Sobel, H. Weiner, V. Kuchroo, and A. Sharpe. 2005. ICOS molecule plays a crucial role in the development of mucosal tolerance. J. Immunol. 175:7341-7347. doi:10.4049/jimmunol.175.11.7341

Molofsky, A., J. Nussbaum, H.-E. Liang, S. Dyken, L. Cheng, A. Mohapatra, A. Chawla, and R. Locksley. 2013. Innate lymphoid type 2 cells sustain visceral adipose tissue 
eosinophils and alternatively activated macrophages. J. Exp. Med. 210:535-549. doi: 10.1084/jem.20121964

Molofsky, A. B., F. Van Gool, H.-E. E. Liang, S. J. Van Dyken, J. C. Nussbaum, J. Lee, J. A. Bluestone, and R. M. Locksley. 2015. Interleukin-33 and Interferon-y counterregulate group 2 innate lymphoid cell activation during immune perturbation. Immunity 43:161-174. doi:10.1016/j.immuni.2015.05.019

Nagakubo, D., O. Yoshie, and T. Hirata. 2016. Upregulated CCL28 expression in the nasal mucosa in experimental allergic rhinitis: Implication for CD4+ memory T cell recruitment. Cell. Immunol. 302:58-62. doi:10.1016/j.cellimm.2016.02.001

Nishioka, T., J. Shimizu, R. lida, S. Yamazaki, and S. Sakaguchi. 2006. CD4+ CD25+ Foxp3+ T cells and CD4+ CD25- Foxp3+ T cells in aged mice. J. Immunol. 176:65866593. doi:10.4049/jimmunol.176.11.6586

O'Brien, C. A., S. J. Batista, K. M. Still, and T. H. Harris. 2019. IL-10 and ICOS differentially regulate $\mathrm{T}$ cell responses in the brain during chronic Toxoplasma gondii infection. J. Immunol. 202:1755-1766. doi:10.4049/jimmunol.1801229

Okamoto, N., K. Tezuka, M. Kato, R. Abe, and T. Tsuji. 2003. PI3-kinase and MAPkinase signaling cascades in AILIM/ICOS- and CD28-costimulated T-cells have distinct functions between cell proliferation and IL-10 production. Biochem. Biophys. Res. Commun. 310:691-702. doi:10.1016/j.bbrc.2003.09.065

Ono, M., H. Yaguchi, N. Ohkura, I. Kitabayashi, Y. Nagamura, T. Nomura, Y. Miyachi, T. Tsukada, and S. Sakaguchi. 2007. Foxp3 controls regulatory T-cell function by interacting with AML1/Runx1. Nature. 446:685-689. doi:10.1038/nature05673

Panneton, V., J. Chang, M. Witalis, J. Li, and W.-K. Suh. 2019. Inducible T-cell costimulator : signaling mechanisms in T follicular helper cells and beyond. Immunol. Rev. 291:91-103. doi:10.1111/imr.12771

Pettersson, U., T. Waldén, P.-O. Carlsson, L. Jansson, and M. Phillipson. 2012. Female mice are protected against high-fat diet induced metabolic syndrome and increase the regulatory T cell population in adipose tissue. PLoS One. 7:e46057.

doi:10.1371/journal.pone.0046057

Rajbhandari, P., B. J. Thomas, A.-C. Feng, C. Hong, J. Wang, L. Vergnes, T. Sallam, B. Wang, J. Sandhu, M. M. Seldin, A. J. Lusis, L. G. Fong, M. Katz, R. Lee, S. G. Young, K. Reue, S. T. Smale, and P. Tontonoz. 2018. IL-10 signaling remodels adipose chromatin architecture to limit thermogenesis and energy expenditure. Cell. 172:218-233. doi: 10.1016/j.cell.2017.11.019.

Redpath, S., N. van der Werf, A. Cervera, A. MacDonald, D. Gray, R. Maizels, and M. Taylor. 2013. ICOS controls Foxp3(+) regulatory T-cell expansion, maintenance and IL- 
10 production during helminth infection. Eur. J. Immunol. 43:705-715. doi:10.1002/eji.201242794

Rivas, M. N., and T. A. Chatila. 2016. Regulatory T cells in allergic diseases. J. Allergy Clin. Immunol. 138:639-652. doi:10.1016/j.jaci.2016.06.003

Sather, B. D., P. Treuting, N. Perdue, M. Miazgowicz, J. D. Fontenot, A. Y. Rudensky, and D. J. Campbell. 2007. Altering the distribution of Foxp3(+) regulatory T cells results in tissue-specific inflammatory disease. J. Exp. Med. 204:1335-1347. doi:10.1084/jem.20070081

Schmitz, J., A. Owyang, E. Oldham, Y. Song, E. Murphy, T. K. McClanahan, G. Zurawski, M. Moshrefi, J. Qin, X. Li, D. M. Gorman, J. F. Bazan, and R. A. Kastelein. 2005. IL-33, an interleukin-1-like cytokine that signals via the IL-1 receptor-related protein ST2 and induces T helper type 2-associated cytokines. Immunity. 23:479-490. doi:10.1016/j.immuni.2005.09.015

Setoguchi, R., S. Hori, T. Takahashi, and S. Sakaguchi. 2005. Homeostatic maintenance of natural Foxp3(+) CD25(+) CD4(+) regulatory T cells by interleukin (IL)-2 and induction of autoimmune disease by IL-2 neutralization. J. Exp. Med. 201:723-735. doi:10.1084/jem.20041982

Smigiel, K. S., S. Srivastava, J. M. Stolley, and D. J. Campbell. 2014a. Regulatory T-cell homeostasis: steady-state maintenance and modulation during inflammation. Immunol. Rev. 259:40-59. doi:10.1111/imr.12170

Smigiel, K. S., E. Richards, S. Srivastava, K. R. Thomas, J. C. Dudda, K. D. Klonowski, and D. J. Campbell. 2014b. CCR7 provides localized access to IL-2 and defines homeostatically distinct regulatory T cell subsets. J. Exp. Med. 211:121-136. doi:10.1084/jem.20131142

So, L., and D. A. Fruman. 2012. PI3K signalling in B- and T-lymphocytes: new developments and therapeutic advances. Biochem. J. 442:465-481. doi:10.1042/BJ20112092

Stolley, J.M., and D. J. Campbell. 2016. A 33D1+ dendritic cell/autoreactive CD4+ T cell circuit maintains IL-2-dependent regulatory T cells in the spleen. J. Immunol. 197:26352645. doi:10.4049/jimmunol.1600974

Tang, Q., K. J. Henriksen, E. K. Boden, A. J. Tooley, J. Ye, S. K. Subudhi, X. X. Zheng, T. B. Strom, and J. A. Bluestone. 2003. Cutting edge: CD28 controls peripheral homeostasis of CD4+CD25+ regulatory T cells. J. Immunol. 171:3348-3352. doi:10.4049/jimmunol.171.7.3348

Vasanthakumar, A., K. Moro, A. Xin, Y. Liao, R. Gloury, S. Kawamoto, S. Fagarasan, L. Mielke, S. Afshar-Sterle, S. Masters, S. Nakae, H. Saito, J. Wentworth, P. Li, W. Liao, 
W. Leonard, G. Smyth, W. Shi, S. Nutt, S. Koyasu, and A. Kallies. 2015. The transcriptional regulators IRF4, BATF and IL-33 orchestrate development and maintenance of adipose tissue-resident regulatory T cells. Nat. Immunol. 16:276-285. doi:10.1038/ni.3085

Vasanthakumar, A., D. Chisanga, J. Blume, R. Gloury, K. Britt, D. C. Henstridge, Y. Zhan, S. V. Torres, S. Liene, N. Collins, E. Cao, T. Sidwell, C. Li, R. G. Spallanzani, Y. Liao, P. A. Beavis, T. Gebhardt, N. Trevaskis, S. L. Nutt, J. D. Zajac, R. A. Davey, M. A. Febbraio, D. Mathis, W. Shi, and A. Kallies. 2020. Sex-specific adipose tissue imprinting of regulatory T cells. Nature. 579:581-585. doi:10.1038/s41586-020-2040-3

Wan, Y. Y., and R. A. Flavell. 2005. Identifying Foxp3-expressing suppressor T cells with a bicistronic reporter. Proc. Natl. Acad. Sci. U.S.A. 102:5126-5131. doi:10.1073/pnas.0501701102

Wan, Z., X. Shao, X. Ji, L. Dong, J. Wei, Z. Xiong, W. Liu, and H. Qi. 2020. Transmembrane domain-mediated Lck association underlies bystander and costimulatory ICOS signaling. Cell \& Mol. Immunol. 17:143-152. doi:10.1038/s41423018-0183-z

Wu, D., A. B. Molofsky, H.-E. E. Liang, R. R. Ricardo-Gonzalez, H. A. Jouihan, J. K. Bando, A. Chawla, and R. M. Locksley. 2011. Eosinophils sustain adipose alternatively activated macrophages associated with glucose homeostasis. Science. 332:243-247. doi:10.1126/science. 1201475

Wu, D., Z. Ren, M. Pae, W. Guo, X. Cui, A. H. Merrill, and S. N. Meydani. 2007. Aging up-regulates expression of inflammatory mediators in mouse adipose tissue. J. Immunol. 179:4829-4839. doi:10.4049/jimmunol.179.7.4829

Wu, D., C. K. Wong, J. M. Han, P. C. Orban, Q. Huang, J. Gillies, M. Mojibian, W. T. Gibson, and M. K. Levings. 2020. T reg-specific insulin receptor deletion prevents dietinduced and age-associated metabolic syndrome. J. Exp. Med. 217:e20191542. doi:10.1084/jem.20191542

Wu, Y. M. Borde, V. Heissmeyer, M. Feuerer, A. Lapan, J. Stroud, D. Bates, L. Guo, A. Han, S. Ziegler, D. Mathis, C. Benoist, L. Chen, and A. Rao. 2006. FOXP3 controls regulatory T cell function through cooperation with NFAT. Cell. 126:375-387. doi:10.1016/j.cell.2006.05.042

Yamaguchi, T., J. B. Wing, and S. Sakaguchi. 2011. Two modes of immune suppression by Foxp3 ${ }^{+}$regulatory T cells under inflammatory or non-inflammatory conditions. Sem. Immunol. 23:424-430. doi:10.1016/j.smim.2011.10.002 
bioRxiv preprint doi: https://doi.org/10.1101/2020.06.01.128504 this version posted February 1, 2021. The copyright holder for this preprint (which was not certified by peer review) is the author/funder, who has granted bioRxiv a license to display the preprint in perpetuity. It is made available under aCC-BY-NC 4.0 International license.

\section{Abbreviations}

$\begin{array}{ll}\text { ATM } & \text { Adipose tissue macrophage } \\ \text { BGL } & \text { Blood glucose level } \\ \text { cT }_{R} & \text { Central regulatory T cell } \\ \text { eT }_{R} & \text { Effector regulatory T cell } \\ \text { HFD } & \text { High-fat diet } \\ \text { ILC2 } & \text { Type 2 innate lymphoid cell } \\ \text { KO } & \text { Icos }^{-/} \\ \text {PI3K } & \text { Phosphoinositide 3-kinase } \\ \text { pLN } & \text { Peripheral lymph node } \\ \text { SQAT } & \text { Subcutaneous adipose tissue } \\ \text { Teff }_{\text {eff }} & \text { Effector T cell } \\ \text { TR }_{R} & \text { Regulatory T cell } \\ \text { VAT } & \text { Visceral adipose tissue } \\ \text { YF } & \text { Icos }^{\text {Y181F }}\end{array}$


A

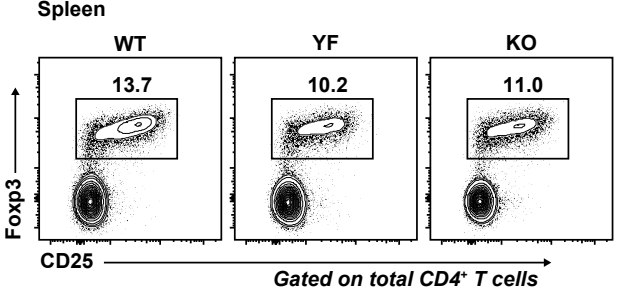

C Spleen

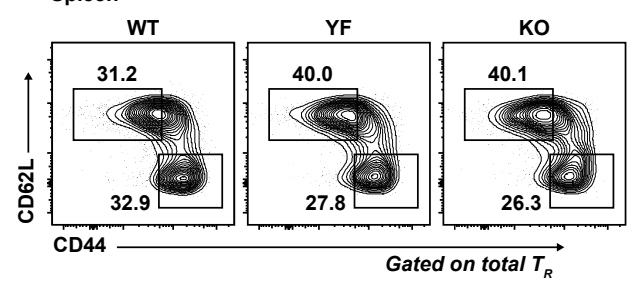

$C T_{R} \quad T_{R}$

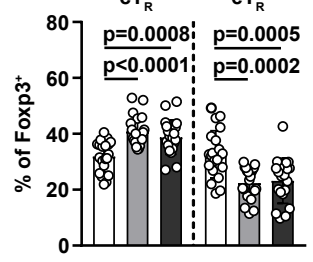
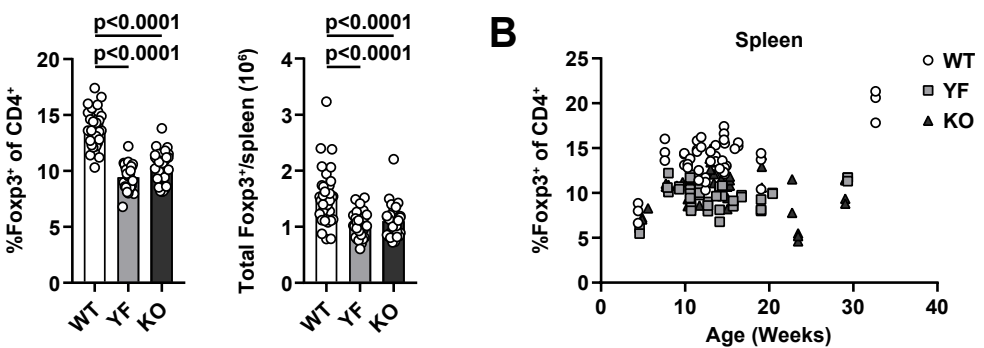

D Spleen
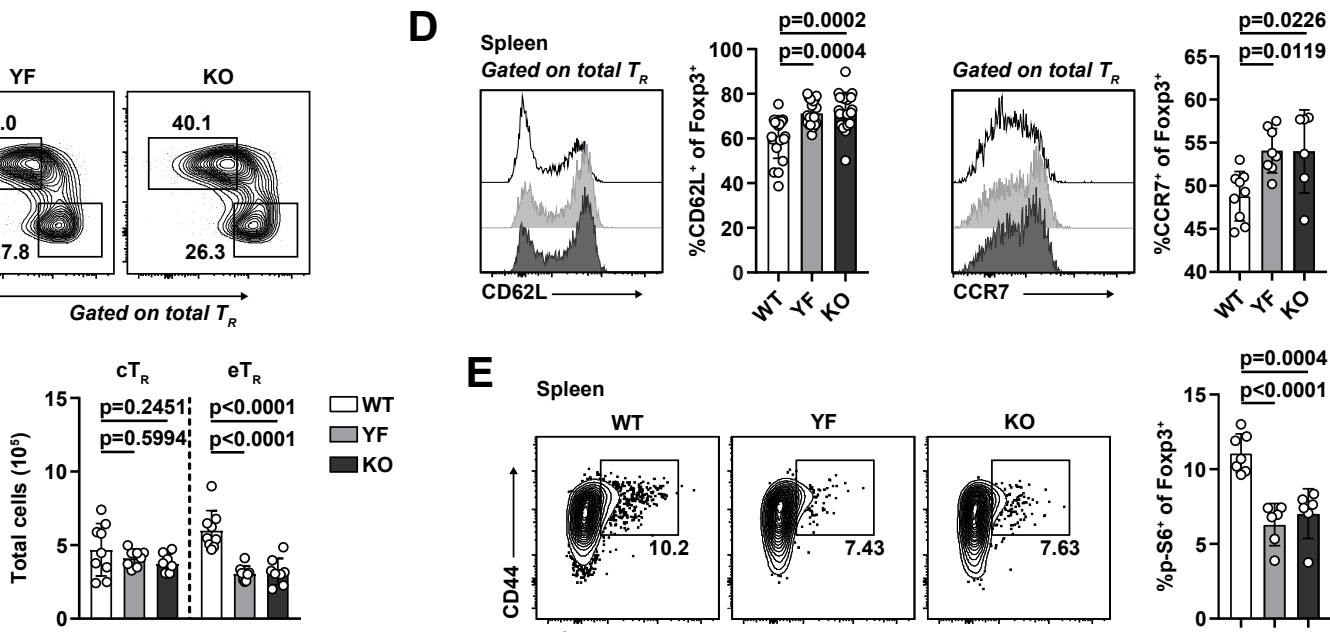

$\mathbf{E}$

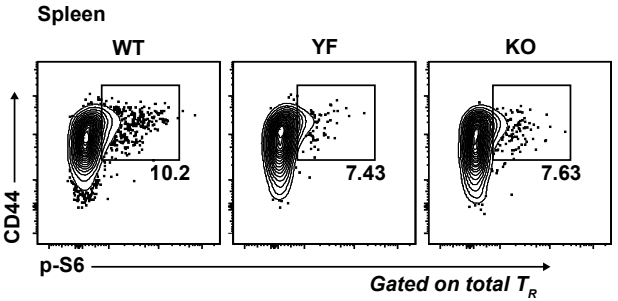

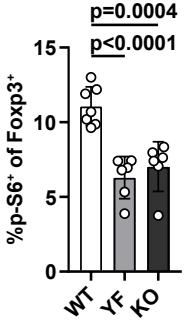


A

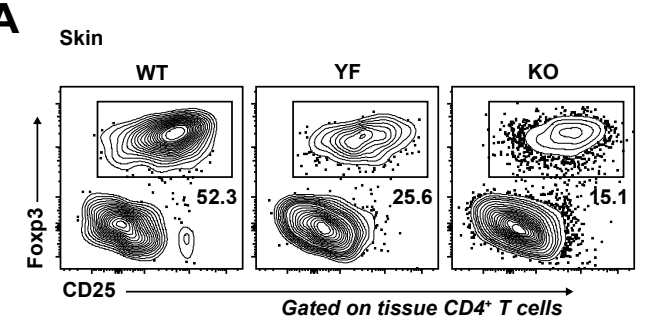

B

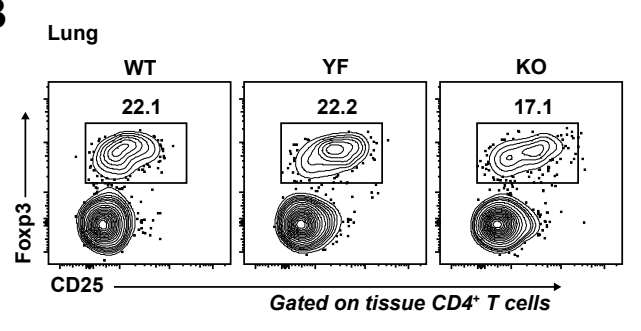

C VAT

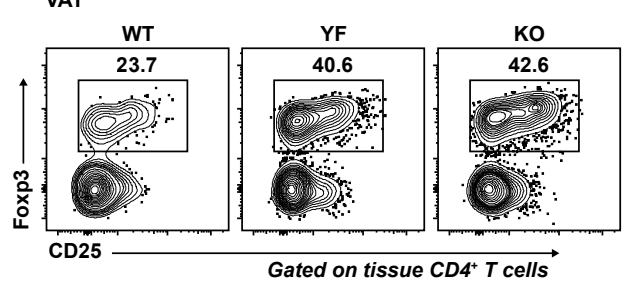

D sqat

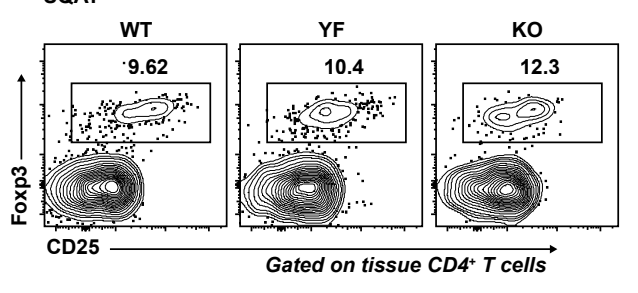

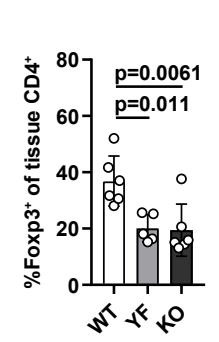
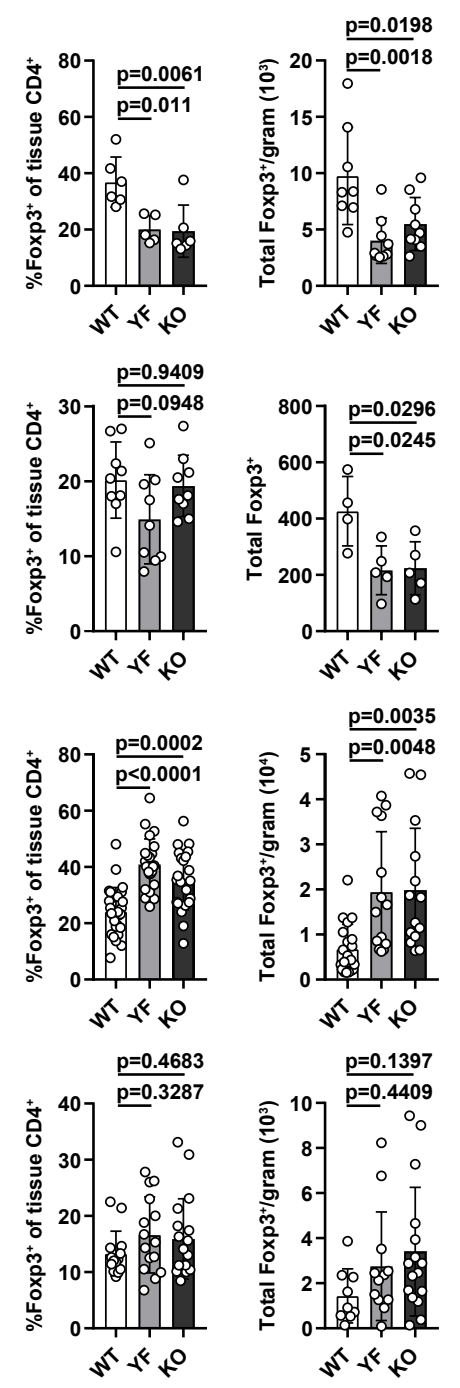

E VAT - Female mice

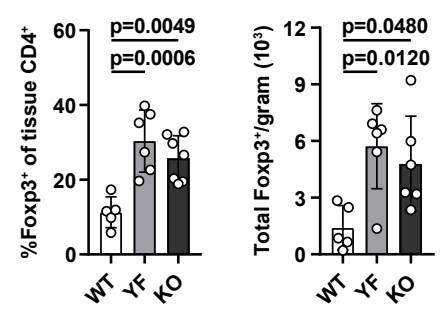

F VAT - WT mice

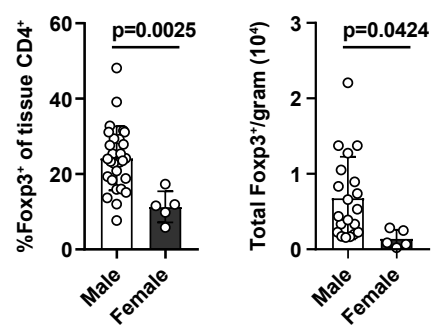

G

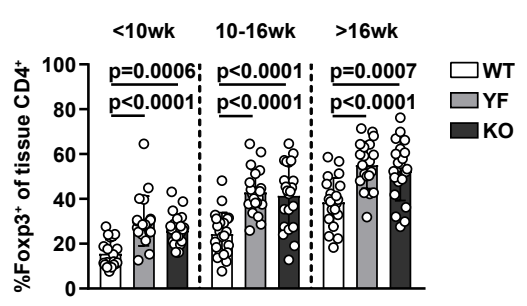


bioRxiv preprint doi: https://doi.org/10.1101/2020.06.01.128504; this version posted February 1, 2021. The copyright halder for this preprint

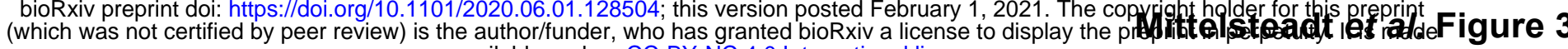
available under aCC-BY-NC 4.0 International license.

A vat
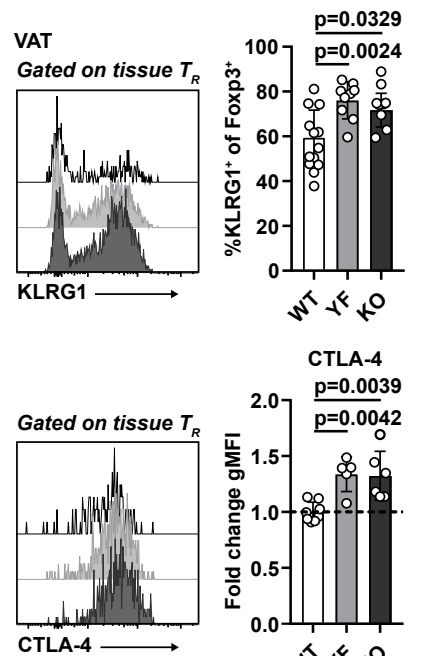

CTLA-4

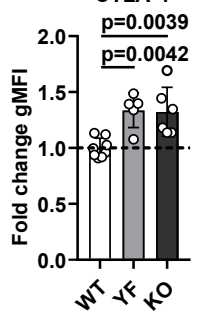

C VAT

Gated on

$\mathrm{CXCR}^{+} \mathrm{ST2}^{+} \mathrm{T}_{\mathrm{R}}$
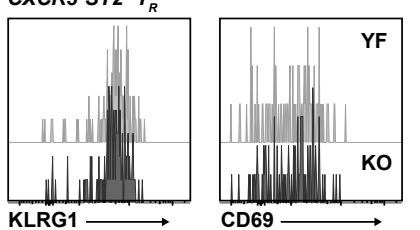

$p=0.0093$

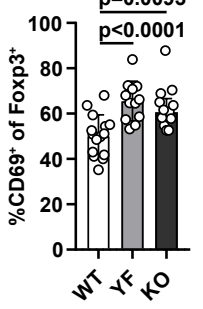

$p=0.0655$

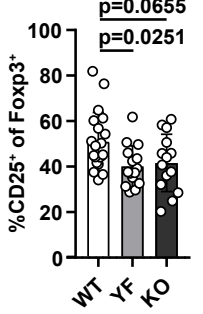

$B$ VAT
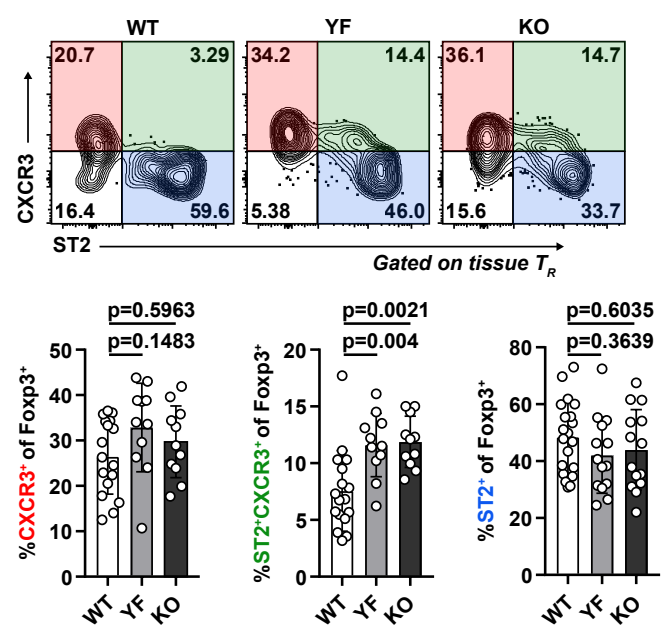

$D_{\text {VAT }}$

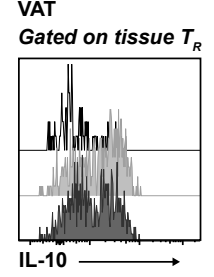

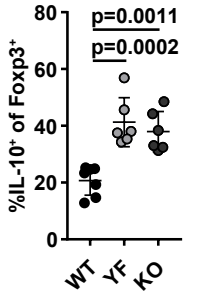

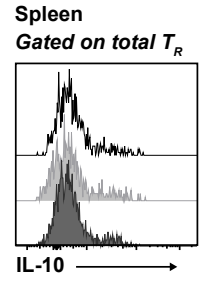

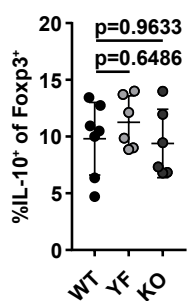



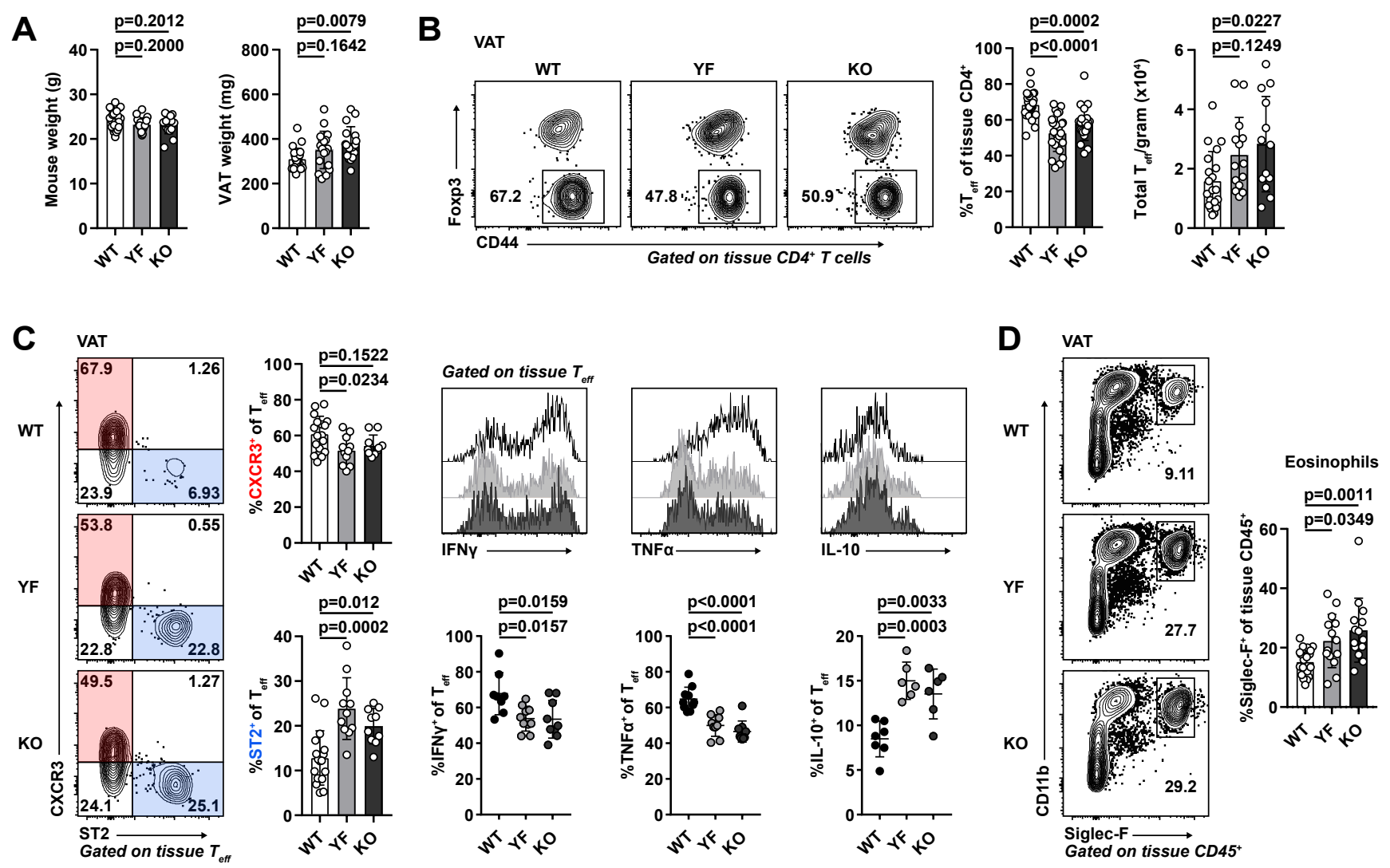
A

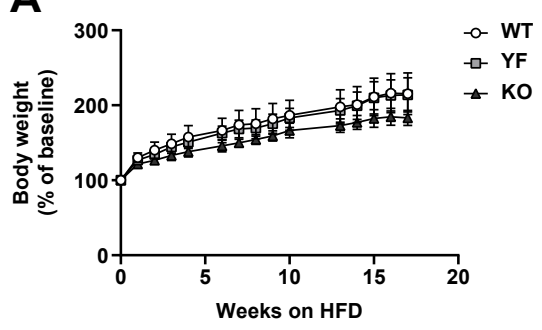

B VAT

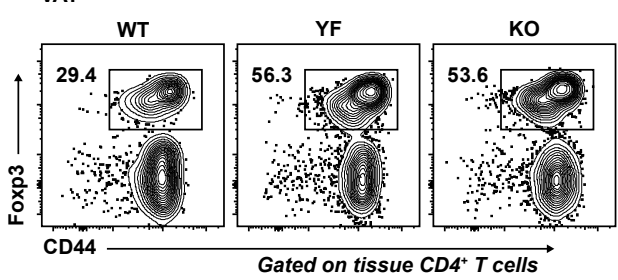

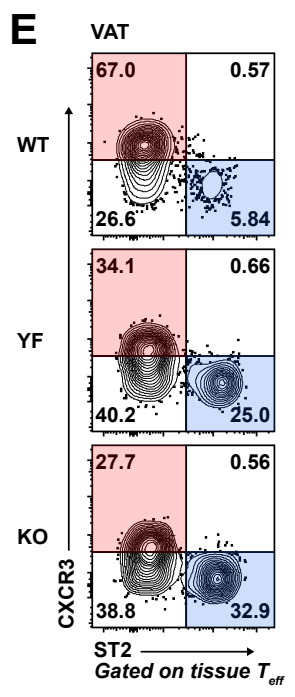
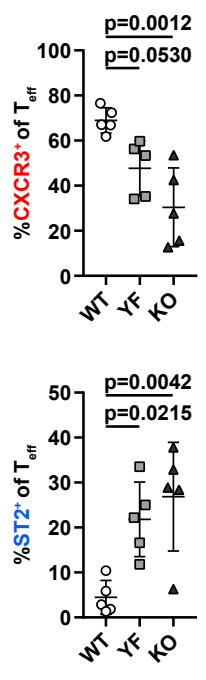
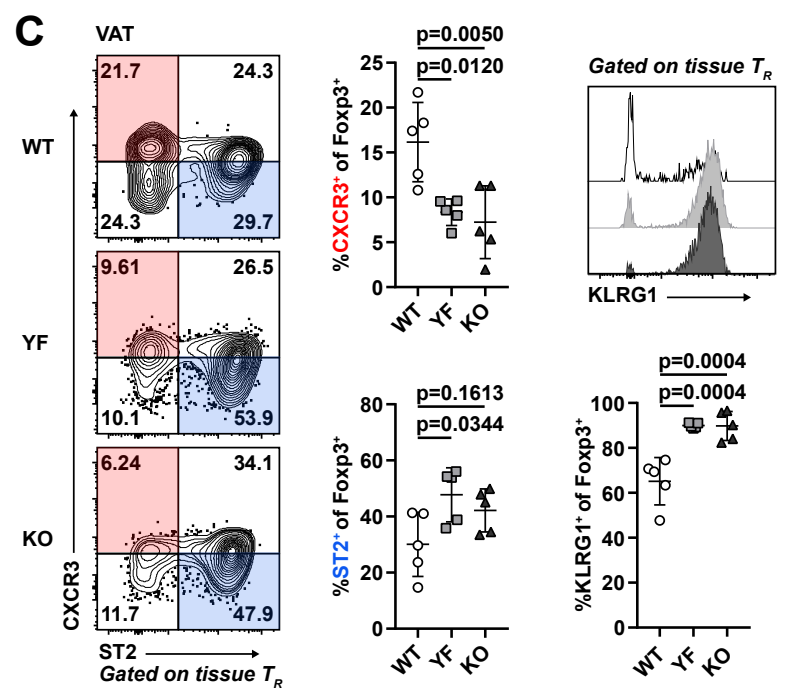

D
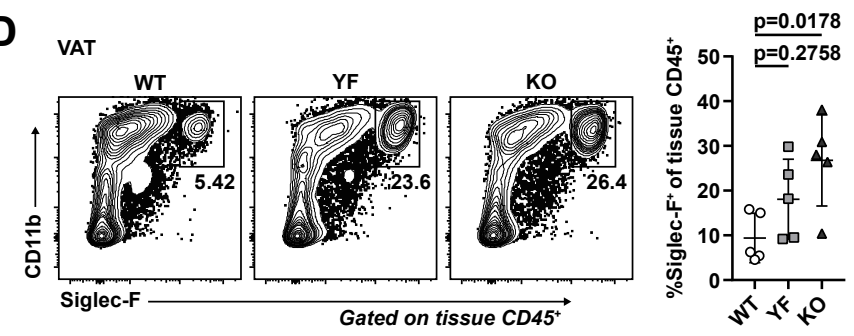

F
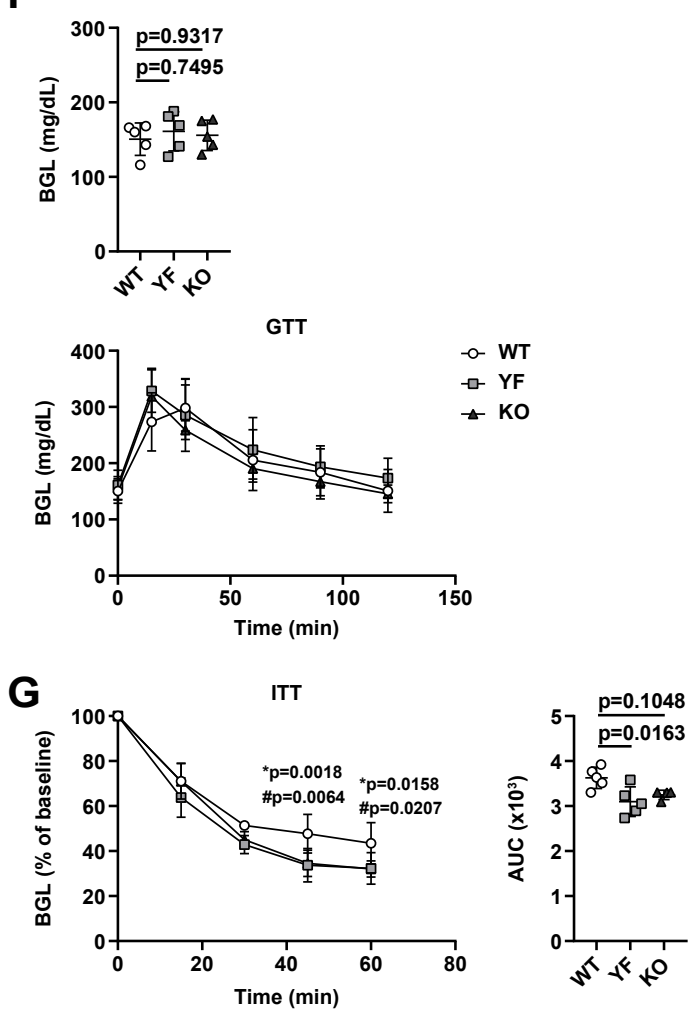


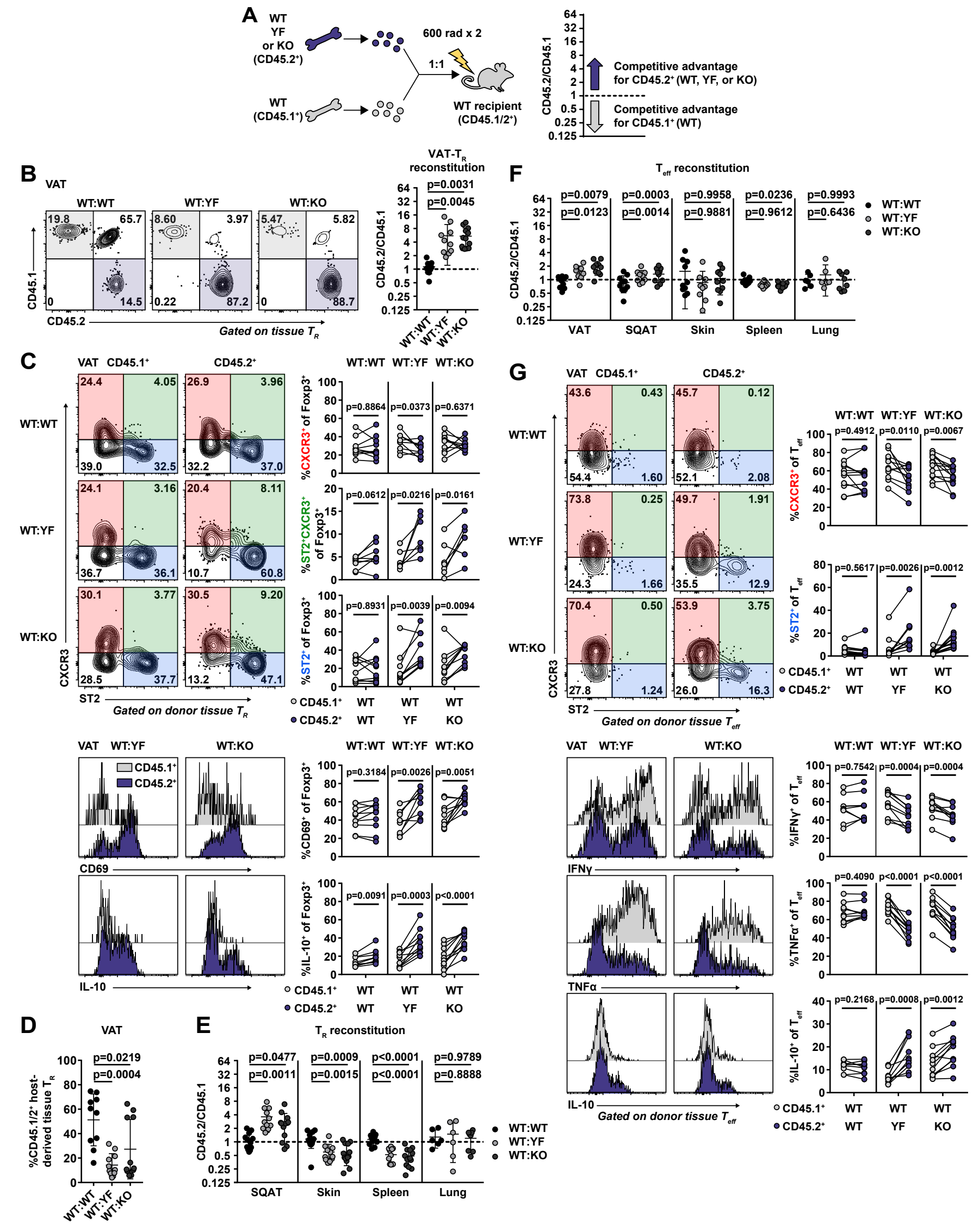


A vat

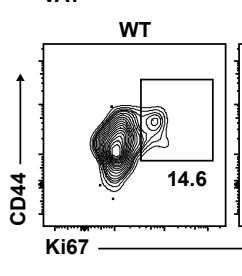

YF

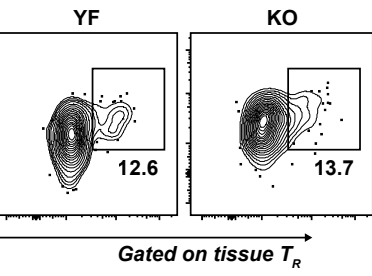

B vaT

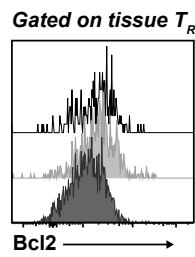

Bcl2

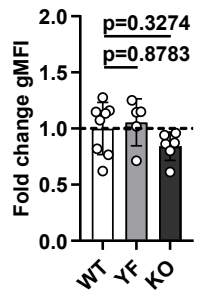

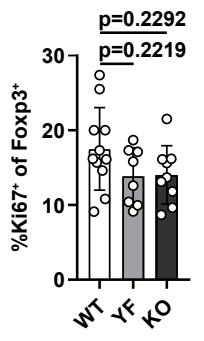


A

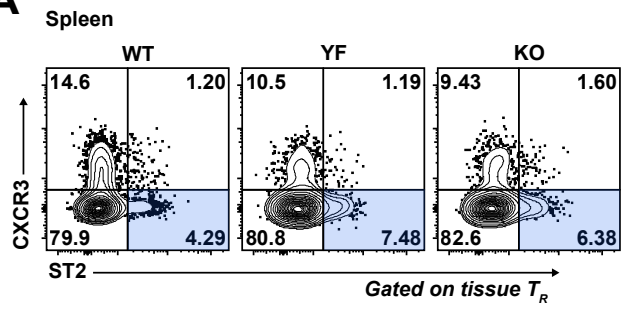

C

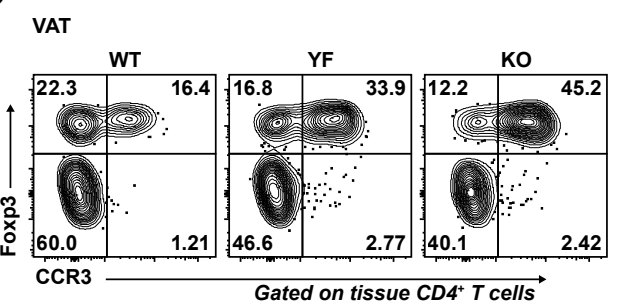

D

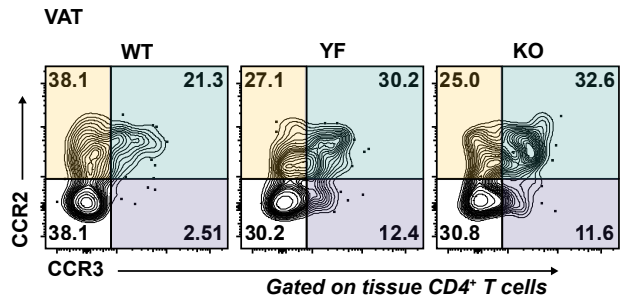

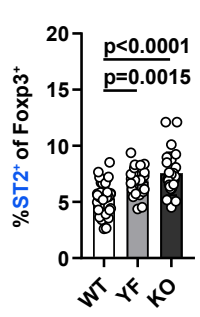

$\leq 8 \mathrm{wk} \quad>8 \mathrm{wk}$ $p=0.0207 \quad p<0.0001$
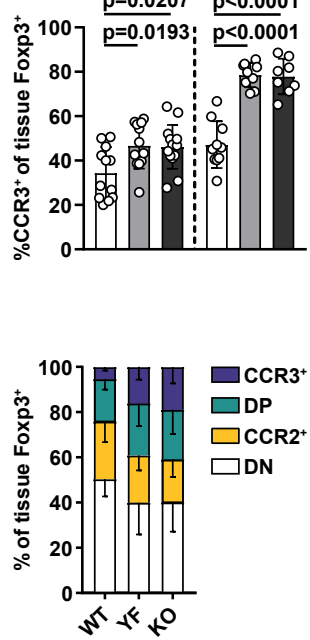

B Spleen - Mixed bone marrow chimeras

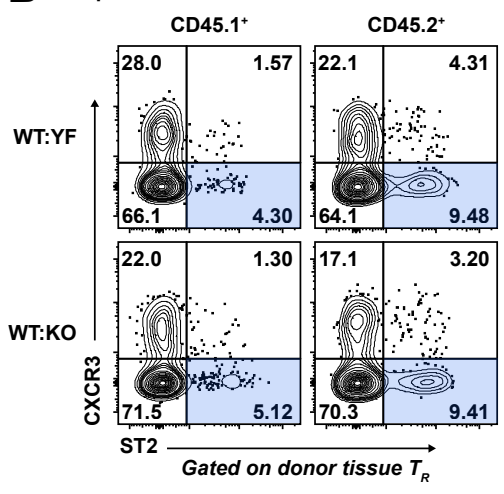

WT:WT WT:YF WT:KO

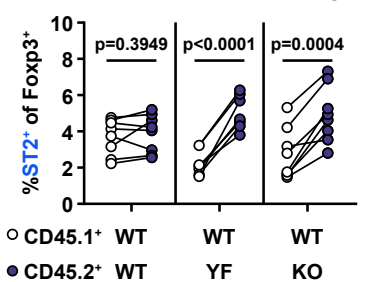

E

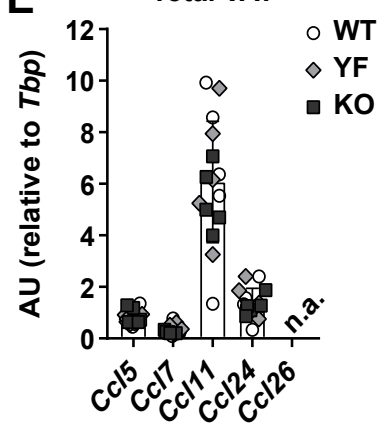

G VAT

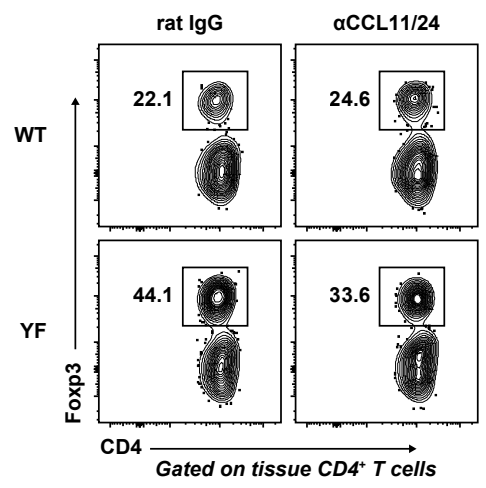

$\mathbf{F}$
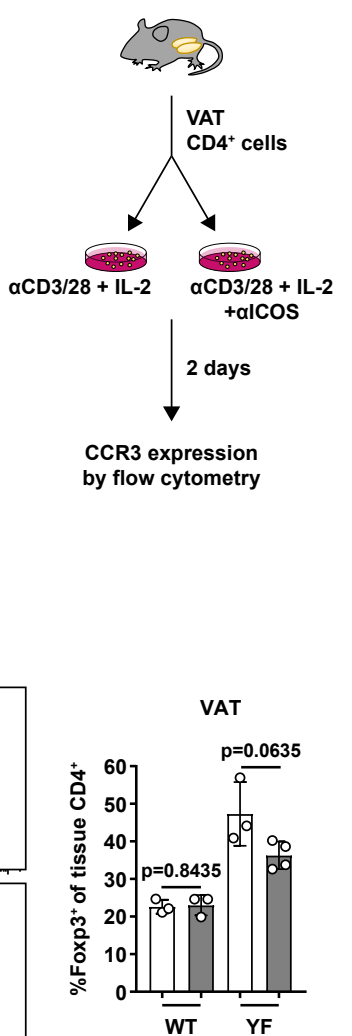
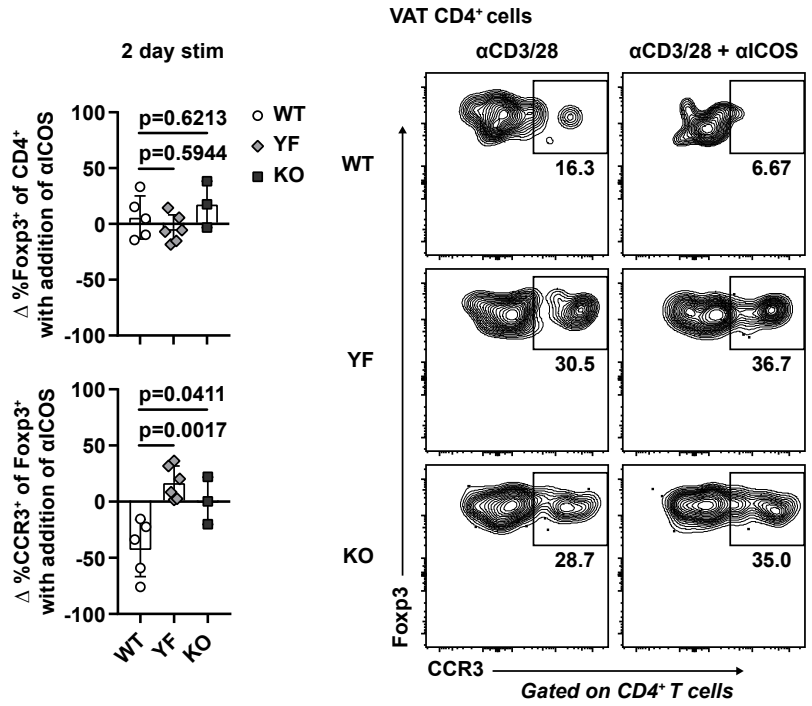

Spleen
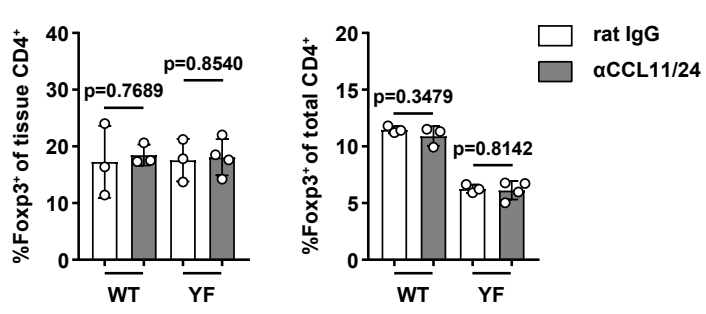